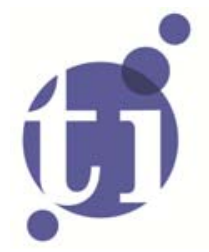

\title{
Historical Developments in Bayesian Econometrics after Cowles Foundation Monographs 10, 14
}

Nalan Basturk ${ }^{1}$

Cem Cakmakli2

S. Pinar Ceyhan'

Herman K. van Dijk1,3

${ }^{1}$ Erasmus School of Economics, Erasmus University Rotterdam, and Tinbergen Institute;

2 Faculty of Economics and Business, University of Amsterdam;

3 Faculty of Economics and Business Administration, VU University Amsterdam. 
Tinbergen Institute is the graduate school and research institute in economics of Erasmus University Rotterdam, the University of Amsterdam and VU University Amsterdam.

More TI discussion papers can be downloaded at http://www.tinbergen.nl

Tinbergen Institute has two locations:

Tinbergen Institute Amsterdam

Gustav Mahlerplein 117

1082 MS Amsterdam

The Netherlands

Tel.: +31(0)205251600

Tinbergen Institute Rotterdam

Burg. Oudlaan 50

3062 PA Rotterdam

The Netherlands

Tel.: +31(0)10 4088900

Fax: $+31(0) 104089031$

Duisenberg school of finance is a collaboration of the Dutch financial sector and universities, with the ambition to support innovative research and offer top quality academic education in core areas of finance.

DSF research papers can be downloaded at: http://www.dsf.nl/

Duisenberg school of finance

Gustav Mahlerplein 117

1082 MS Amsterdam

The Netherlands

Tel.: +31(0)20 5258579 


\title{
Historical Developments in Bayesian Econometrics after Cowles Foundation Monographs 10, 14*
}

\author{
Nalan Basturk ${ }^{1,5}$, Cem Cakmakli ${ }^{2,4}$, S. Pinar Ceyhan ${ }^{1,5}$, and \\ Herman K. van Dijk ${ }^{1,3,5}$ \\ ${ }^{1}$ Econometric Institute, Erasmus University Rotterdam \\ ${ }^{2}$ Department of Quantitative Economics, University of Amsterdam \\ ${ }^{3}$ Department of Econometrics, VU University Amsterdam \\ ${ }^{4}$ Department of Economics, Koc University \\ ${ }^{5}$ Tinbergen Institute
}

November 2013

\begin{abstract}
After a brief description of the first Bayesian steps into econometrics in the 1960s and early $70 \mathrm{~s}$, publication and citation patterns are analyzed in ten major econometric journals until 2012. The results indicate that journals which contain both theoretical and applied papers, such as Journal of Econometrics, Journal of Business and Economic Statistics and Journal of Applied Econometrics, publish the large majority of high quality Bayesian econometric papers in contrast to theoretical journals like Econometrica and the Review of Economic Studies. These latter journals published, however, a few papers that had a substantial impact on Bayesian research. The journals Econometric Reviews and Econometric Theory published key invited papers and special issues that received wide attention, while Marketing Science shows an ever increasing number of papers since the middle nineties. The International Economic Review and the Review of Economics and Statistics show a moderate time varying increase. The early nineties indicate an upward movement in publication patterns in most journals probably due to the effect of the 'Computational Revolution'. Next, a visualization technique is used to connect papers and authors around important theoretical and empirical themes such as forecasting, macro models, marketing models, model uncertainty and sampling algorithms. The information distilled from this analysis shows the names of authors who contribute substantially to particular themes. This is followed by a discussion of those topics that pose interesting challenges for discussion amongst Bayesian econometricians, namely the computational revolution, unobserved component and flexible model structures, choice models, IV models, dynamic models and forecasting. Three issues are summarized where Bayesian and frequentist econometricians differ: Identification, the value of prior information and model evaluation; dynamic inference and nonstationarity; and vector autoregressive versus structural modeling. A major topic of debate amongst Bayesian econometricians is listed as objective versus subjective econometrics and communication problems and bridges between statistics and econometrics are summarized. The paper ends with a list of four important themes that will be a challenge for twenty-first century Bayesian econometrics: Sampling methods which are suitable for parallelization and GPU calculations, complex economic models which can account for nonlinearities, analysis of implied model features such as risk and instability and incorporating model incompleteness in econometric analysis.
\end{abstract}

Keywords: History, Bayesian Econometrics

JEL Classification: B4, C1

*Nalan Basturk (basturk@ese.eur.nl), Cem Cakmakli (C.Cakmakli@uva.nl), S. Pinar Ceyhan (ceyhan@ese.eur.nl), correspondence to Herman K. van Dijk (hkvandijk@ese.eur.nl). A preliminary version of this paper was presented at the NBER Workshop on Methods and Applications for DSGE Models at the Philadelphia Federal Reserve Bank, September 2013. Useful comments from Rui Almeida, Lennart Hoogerheide and Frank Schorfheide are gratefully acknowledged. Nalan Basturk and Herman K. van Dijk are supported by NWO Grant 400-09-340 and Cem Cakmakli by the AXA Research Fund. 


\section{Introduction}

Bayesian econometrics is now widely used for inference, forecasting and decision analysis in economics, in particular, in the fields of macro-economics, finance and marketing. Three practical examples are: International corporations that sell their goods abroad want to know the risk of foreign exchange rate exposure that they incur at the time they repatriate the proceeds of their sales, see Bos, Mahieu, and Van Dijk (2000); in modern macroeconomics the risk of a liquidity trap, defined as low inflation, low growth and an interest rate close to the zero lower bound, is relevant for an adequate economic policy for several countries; evaluating the effect of a new pricing policy is highly relevant in decision strategies of supermarket chains. Particular references and more examples are given in textbooks like Geweke (2005); Rossi et al. (2005) and Koop et al. (2007). This widespread interest in and use of empirical probability assessments of important economic issues has come a long way from the early steps of Bayesian econometrics in the 1960s following the likelihood based inference reported in the brilliant Cowles Foundations monographs 10 and 14, see Koopmans (1950) and Hood and Koopmans (1953). Papers in these monographs applied the likelihood approach introduced by R.A. Fisher (Fisher, 1912, 1922) to, predominantly, a system of simultaneous equations where immediate feedback mechanisms or, otherwise stated, a set of jointly endogenous variables posed substantial methodological challenges to estimate systems of equations. In the early and middle part of the 1970s there were several shocks to this line of research. Data series exhibited novel features like strong persistence and time varying volatility; new modeling like the vector autoregressive approach was developed and novel simulation based inferential techniques based on Importance Sampling and Markov chain Monte Carlo were introduced. This opened a wide set of new research lines that had substantial methodological and practical consequences for economic analysis, forecasting and decision strategies. Structural economic models based on dynamic stochastic general equilibrium concepts, unobserved component models allowing for time varying parameters and using data augmentation, simulation methods and increased focus on the complete forecast distribution and in particular in the tails of the distribution like in Value-at-Risk are only a few examples in this respect.

In the present paper we sketch historical developments of Bayesian econometrics from the early 1960s until 2012 by collecting and analyzing the publication and citation patterns on Bayesian econometric papers in ten major econometric journals until 2012. The number of pages written on Bayesian econo- 
metrics were recorded as a percentage of total number of pages for each year for all journals. The results indicate that journals which contain both theoretical and applied papers, such as Journal of Econometrics, Journal of Business and Economic Statistics and Journal of Applied Econometrics, publish the large majority of high quality Bayesian econometric papers in contrast to theoretical journals like Econometrica and the Review of Economic Studies. These latter journals published, however, some high quality papers that had a substantial impact on Bayesian research. The journals Econometric Reviews and Econometric Theory published key invited papers and/or special issues that received wide attention, while Marketing Science shows an ever increasing number of papers since the middle nineties. The International Economic Review and the Review of Economics and Statistics show a moderate time varying increase. It is noteworthy that since the early nineties there exists a upward movement in publication patterns in most journals probably due to the effect of the 'Computational Revolution'.

Next, a visualization technique is used to connect papers and authors around important themes in theoretical and empirical econometrics. The proximity of topics that we consider is defined by the number of times that the keywords or names appear together or in relation to all keywords and names cited together. The results show the interconnectedness of several topics of interest. Macroeconomics and finance literature is related to simulation and filtering methods as well as methods dealing with model uncertainty. Macro models used for policy purposes are related to identification issues. Marketing models are linked to flexible model and prior structures such as hierarchical Bayes and Dirichlet processes. The information distilled from this analysis shows also names of authors who contribute substantially to particular themes. This is followed by a discussion of those topics that pose interesting challenges for discussion amongst Bayesian econometricians. The effects of the computational revolution on Bayesian econometrics, advances in several fields, such as flexible and unobserved component model structures, dynamic models and forecasting, the issue of identification and prior information, as well as three main debates between Bayesian and frequentist econometricians are presented. There is probably not a subject area in the literature where everything goes smoothly. Bayesian econometrics is no exception. We only summarize major debates and issues encountered in the Bayesian econometrics literature in the latter part of the twentieth century. The paper ends with a list of important themes that are predicted to be a challenge for twenty-first Bayesian econometrics. These refer to big data, model complexity, parallel computing and model incompleteness.

Influential papers in Bayesian econometrics have been analyzed in Poirier $(1989,1992)$, which pro- 
vide quantitative evidence of the impact of the Bayesian viewpoint as measured by the percentage of pages devoted to Bayesian topics in leading journals. We contribute to this literature by extending the bibliographical data with more recent papers and additional leading journals. Our contribution differs from the literature in several ways. First, regarding the influential papers in the field, we consider an alternative measure, the number of citations of each paper in addition to the percentage of pages devoted to Bayesian topics. The impact of papers are found to be different according to the criteria chosen for this purpose. Second, we define the set of influential papers in the field relying on the references in Geweke, Koop, and Van Dijk (2011), who provide an up-to-date set of references in the field with their respective subfields, such as macroeconomics and computational advances. Third, we consider the clustering of papers in the field without defining a measure for their influence. This analysis is based on online bibliographic databases, and the results are not affected by the subjective definition of influential papers in the field of Bayesian econometrics.

The paper is organized as follows: In Section 2 some first Bayesian steps into the field of econometrics are listed. Section 3 analyzes the publication and citation patterns of the papers in our database. Section 4 visualizes the connectivity of the topics in those papers, linking these to the key topics of interest during the period 1962-2012. Section 5 is devoted to several issues and debates that are pivotal in the history of Bayesian econometrics. Section 6 contains the authors' expectations about the future of Bayesian econometrics and concludes. We note that many more interesting Bayesian empirical papers exist and are published in major economics journals. These are, however, outside the scope of the present paper.

\section{Cowles Foundation Research and Early Bayesian Econometric Steps}

The Cowles Foundation Monographs 10 and 14, published in 1950 and 1953 respectively, laid the foundations for modern inference in econometrics after the famous Haavelmo paper (Haavelmo, 1944) on the probability approach to econometrics. Although Haavelmo listed two interpretations of probability: the 'frequentist' concept and the 'a priori confidence' one, see Haavelmo (1944) pp. 48, the first one was use henceforth in econometrics. The focus in Monographs 10 and 14 was on developing the method of maximum likelihood for systems of simultaneous equations. Identification issues, full information maximum likelihood, limited information maximum likelihood, the corresponding numerical optimization methods to find the maximum and also some basic time series problems were analyzed. Among the contributors to 
this area are Koopmans (1945), Anderson (1947), Anderson and Rubin (1949), Hurwicz (1950), Chernoff and Divinsky (1953) and Chernoff (1954).

Much of this research followed the likelihood approach from R.A. Fisher. Although Fisher rejected Bayesianism, he had an alternative: so-called fiducial inference (see Fisher (1973) and Aldrich (1995) for a review), which was Fisher's attempt to use inverse probability and to analyze the shape of the likelihood without stochastic prior information. This has been characterized by Savage as: 'A bold attempt to make the Bayesian omelette without breaking the Bayesian eggs' (Savage, 1961).

The frequentist interpretation of estimators obtained by using the likelihood approach became known as the 'classical approach' in econometrics. As argued, in Cowles Foundation Monograph 23, by Rothenberg, this classical approach has some very restrictive assumptions, see Rothenberg (1973). First, for efficiency, accuracy, and credibility one usually makes use of the Crámer-Rao lower bound of the variance of the estimator. This holds only for unbiased estimators and is in most cases only asymptotically valid. Second, one makes use of prior conditioning information as exact restrictions, which is often unrealistic and overly restrictive. Third, this ignores completely the decision aspect of inference.

Given all the work on the implementation of the likelihood approach to econometrics and the recognition of its limitations, it is very natural that the Bayesian approach would follow. ${ }^{1}$ Among early contributions to this literature are three important books. The first one is Raiffa and Schlaifer (1961) who introduced the concept of conjugate analysis as a way to construct informative prior information on model parameters. The idea is that the model was already in existence in the period before the data were observed or alternatively that the model was in existence for related data sets in other countries or for a different set of agents with similar features. A second book was Schlaifer (1959), later summarized in Pratt et al. (1995), where practical decision problems were explained and analyzed. Here a connection with the field of finance and business was made. Thirdly, there came the very influential 'Bible' of analytical results in Bayesian econometrics by Zellner (1971). All econometric models that were in use at that time were analyzed in this classic book from a Bayesian perspective. Analogies and differences between the classical and Bayesian approach were discussed, often using a weak or non-informative prior approach.

Following these early Bayesian steps, there were several issues that attracted attention in the litera-

\footnotetext{
${ }^{1}$ Note that in post World War-II econometrics one has, apart from the likelihood approach, the (dynamic) regression methods and GMM as major schools of econometric inference. We only refer to the likelihood approach in the present paper.
} 
ture. We document four of these issues in this section.

\section{Rothenberg's problem and natural conjugate priors}

The natural conjugate family gives an analytically tractable and convenient family of prior densities for the case of the standard linear regression model. This density is known as the normal-inverted gamma density and it allows to update prior information using Bayes theorem in a simple way: The posterior mean of the regression parameters is a weighted average of prior mean and data mean with weights that are given as the relative accuracy of prior and data mean, respectively.

However, for a system of regression equations the corresponding conjugate prior has an important restriction on the prior variances of the regression equations parameters. This can be shown as follows. Consider the system

$$
Y=X \Pi+V
$$

where $Y$ and $X$ are appropriate data matrices from $T$ observations and $\Pi$ is a parameter matrix and the columns of $V$ are $\operatorname{NID}(0, \Sigma)$ where NID denotes the independent normal distribution. As shown in Rothenberg (1963), the likelihood of $(\Pi, \Sigma \mid Y, X)$ can be written as

$$
f(\Pi, \Sigma \mid Y, X) \propto|\Sigma|^{-\frac{T}{2}} \exp \left(-\frac{1}{2} \operatorname{tr} \Sigma^{-1}\left((\Pi-P)^{\prime}\left(X^{\prime} X\right)(\Pi-P)+T S\right)\right),
$$

where $P$ and $S$ are the maximum likelihood estimators: $P=\left(X^{\prime} X\right)^{-1} X^{\prime} Y, S=\frac{1}{T}(Y-X P)^{\prime}(Y-X P)$.

A prior density that belongs to the conjugate family and that is proportional and of the same functional form as the likelihood in (2) implies that the variance of $\pi_{i j}$ is given as

$$
\operatorname{Var}\left(\pi_{i j}\right)=\sigma_{i i} a_{j j}
$$

where $\sigma_{i i}$ is the $i$ th diagonal element of $\Sigma$ and $a_{j j}$ is the $j$ th diagonal element of $\left(X^{\prime} X\right)^{-1}$.

It follow that one has the restriction

$$
\frac{\operatorname{Var}\left(\pi_{r i}\right)}{\operatorname{Var}\left(\pi_{s i}\right)}=\frac{\operatorname{Var}\left(\pi_{r j}\right)}{\operatorname{Var}\left(\pi_{s j}\right)}
$$

that is, the variances of the parameters in the $r^{\text {th }}$ equation have to be proportional to the variances 

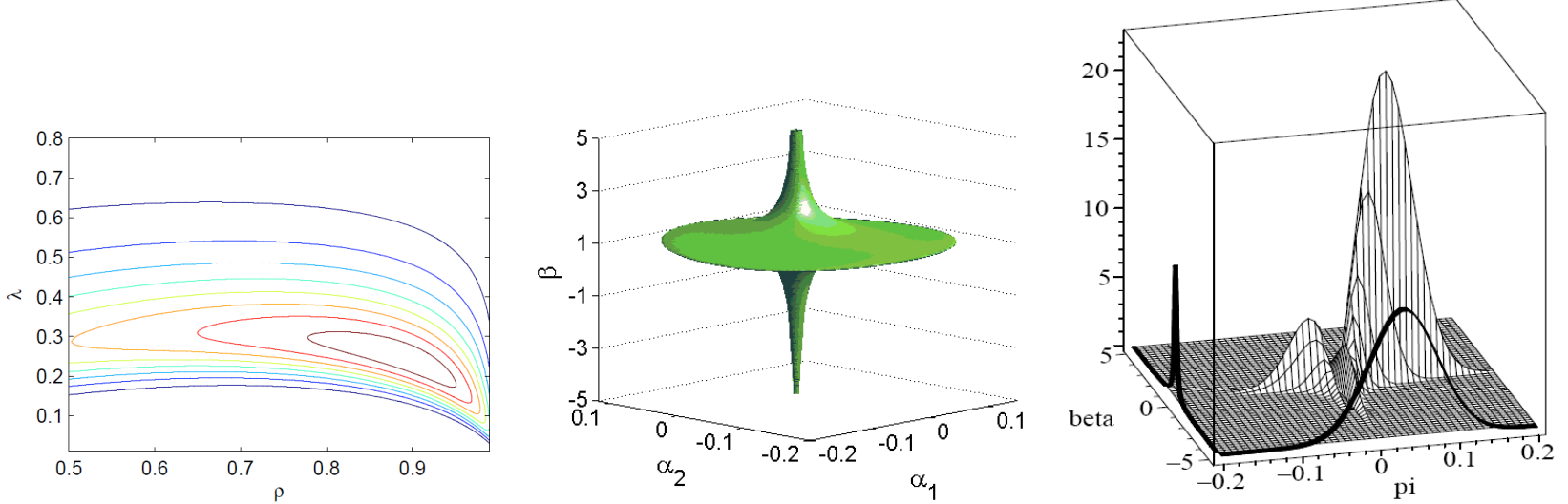

of the corresponding parameters in the $s^{\text {th }}$ equation. There is no a priori economic reason why this mathematical restriction should be the case. This creates a problem for inference in systems of equations, like Seemingly Unrelated Regression Equations (SURE) and Vector AutoRegressive (VAR) models, and it is known as 'Rothenberg's problem', see Rothenberg $(1963,1973)$.

In further research Drèze and Richard followed a path to limit this restriction by either concentrating the analysis on a single equation within a system of equations which is known as the Bayesian limited information approach, see Drèze (1976) and Bauwens and Van Dijk (1990) for details, or by extending the natural conjugate family for a system of equations, see Drèze and Richard (1983). However cavalier these approaches were, the restriction on the natural conjugate prior family is a severe one and different attempts in research were started to free the analysis from analytical restrictions.

\section{Monte Carlo gives freedom from analytical restrictions and allows for evaluation of uncer- tainty of policy effectiveness}

Monte Carlo simulation has freed the Bayesian approach from very restrictive model structures: It allows Bayesians to apply their inference to a wide range of complex models in many scientific disciplines. Figure 1 shows examples of complex (non-elliptical) posterior distributions in models for realistic problems in economics. The posterior distributions occur in finance (modeling daily stock returns), macroeconomics (modeling the joint behavior of variables with a long run equilibrium relationship), and microeconomics (modeling the effect of education on income), see Hoogerheide, Opschoor, and Van Dijk (2012) and Basturk, Hoogerheide, and Van Dijk (2013b).

Using novel simulation methods one can obtain reliable and accurate estimates of the properties of 
interest of such posterior distributions. Importance Sampling, introduced into statistics and econometrics by Kloek and Van Dijk (1975) and later published as Kloek and Van Dijk (1978), or the independence chain Metropolis-Hastings algorithm, Metropolis et al. (1953) and Hastings (1970), can be used.

Apart from this need to free the Bayesian approach from restrictive priors, there also existed interest in evaluating uncertainty of policy effectiveness. An important example was to obtain the posterior distribution of the multiplier in a system of simultaneous equations, see Brainard (1967). Here one faces the issue that this multiplier is usually a ratio or more general a rational function of structural parameters. The Monte Carlo method approach gives here an easy operational approach to obtain the finite sample distribution, see e.g. Van Dijk and Kloek (1980).

\section{Testing, signifying nothing, sequential testing and credibility of the final chosen model}

The focus on statistical testing in econometrics in the past fifty years has not yielded substantial confidence in obtained results. The focus on statistical testing regularly means that the researcher does not raise the issue whether the results matter from an economic point of view. Statistical significant but economically almost meaningless is something that decision makers will not accept as a sound basis for policy analysis, see McCloskey and Ziliak (1996) and The Economist (2004). A more fundamental statistical weakness of the 'classical approach' is the testing of many different hypotheses in econometric models by a sequential testing procedure. In the analysis it is usually not taken into account that the distribution of the second test depends on the outcome of the first one and so on for further tests. In the end a model is 'accepted' without stating a measure of 'credibility' of the final result. A natural Bayesian solution is to give weights to particular model features by using Bayesian model averaging and pursue forecasting with a weighted average of model structures. This line of research is shown in, e.g. Wright (2008) and Strachan and Van Dijk (2013).

\section{Conditional probability statements on features of interest}

Another fundamental problem with the classical approach is the difficulty of dealing with the issue of conditional probability statements which is a concept that is widely used in practice. Given a set of data decision makers are usually interested in the probability of an unknown feature of a problem at hand. The earlier listed examples are clear indications: How to hedge currency risk for international corporations given data on exchange rate behavior; What monetary policy to be used in the face of a 
liquidity trap given data for countries like Japan and the European Union; and Which advertising policy given scanner data about customer behavior are relevant. The simulation based Bayesian approach is very suitable for such conditional probability statements.

These issues will be dealt with in more detail in section 5 .

\section{Exploratory Data Analysis}

In this section we analyze the advance of Bayesian econometrics since the middle of the 1970s from a descriptive point of view. Specifically, we analyze how Bayesian econometrics got in the mainstream and high quality econometric journals using the publication and citation patterns of 952 papers in leading journals during the period between 1978 and 2012. We select these papers on the basis of their contributions to theoretical or applied topics in Bayesian econometrics and denote them by 'Bayesian papers'. The list of leading journals consists of 10 journals: Econometrica (Ectra), Econometric Reviews (ER), Econometric Theory (ET), International Economic Review (IER), Journal of Applied Econometrics (JAE), Journal of Business and Economic Statistics (JBES), Journal of Econometrics (JE), Marketing Science (MS), Review of Economic Studies (RES) and Review of Economics and Statistics (ReStat). Our analysis extends the one from Poirier (1992) by including more journals and a longer period. We also make use of citation patterns. Detailed statistics of the papers considered in this section are provided in Appendix A, Tables A.1, A.2, A.3 and A.4.

\subsection{Publication Patterns}

The first criterion we use to analyze the advances in Bayesian econometrics is the percentages of Bayesian pages in the leading econometrics and quantitative economics journals that were listed above.

The top panel in Figure 2 presents the annual percentages of the pages allocated to Bayesian papers for each journal. These percentages are usually below 30\%, with exceptions in ER, ET and MS. There are only two journal issues which have more than 40\% Bayesian content. The ER issue in 1984 has four Bayesian papers constituting $44.93 \%$ of the total number of pages with as most influential paper the one by Doan et al. (1984). The 2007 issue of ER also has a high percentage of Bayesian pages, where $56.83 \%$ of the issue is devoted to 18 Bayesian papers, including An and Schorfheide (2007) as one of the largest papers. 
Special issues yield the highest values reported in the top panel of Figure 2. These are the ER issues in 1984, 1999, 2007; the ET issue of 1994 (on Bayes methods and unit roots); the JE issue in 1991, and to a lesser extent the ones in 1985, 2004, and 2012.

The bottom panel in Figure 2 presents the average percentage of pages for Bayesian papers in each journals over 5-year intervals. These average percentages provide general publication patterns compared to the top panel of Figure 2 since the influence of special journal issues related to Bayesian econometrics is now more limited due to the 5-year averaging.

The bottom panel of Figure 2 shows that the influence of Bayesian econometrics in terms of the percentage of allocated pages is time varying and journal dependent. Journals such as Ectra and IER typically have low percentages of Bayesian pages. On the other hand, JBES, JAE and MS typically have high percentages of Bayesian pages, with a substantial increase in these percentages after 1990s. The set of journals with a large share of Bayesian papers show that Bayesian inference is mainly present in applied papers rather than theoretical papers. Figure 2 indicates two main clusters of journals in terms of their focus on Bayesian econometrics. The first cluster consists of journals with relatively low average number of percentages of Bayesian pages: Ectra, ET, IER, RES and ReStat. The average percentages of Bayesian pages in these journals are less than $5 \%$. The second cluster consists of journals with relatively high average percentages of Bayesian pages: ER, JAE, JBES, JE and MS. The increased share of Bayesian pages is most visible for MS, then for ER and JAE, particularly for the period after 1992. To a lesser extent, this increasing pattern holds for Ectra, IER and RES. This general increasing influence of Bayesian econometrics after 1990s can be attributed to computational advances making Bayesian inference easier and the increased number of applied papers using Bayesian inference.

In summary, a high cluster of published Bayesian papers and a low cluster appear, where the low cluster refers to the more theoretical journals. Special issues contain more Bayesian papers and a structural break, indicating an increasing number of Bayesian papers, occurs since the early 1990s due the use of novel computational techniques. 
Figure 2: Percentages of pages allocated to Bayesian papers for all journals
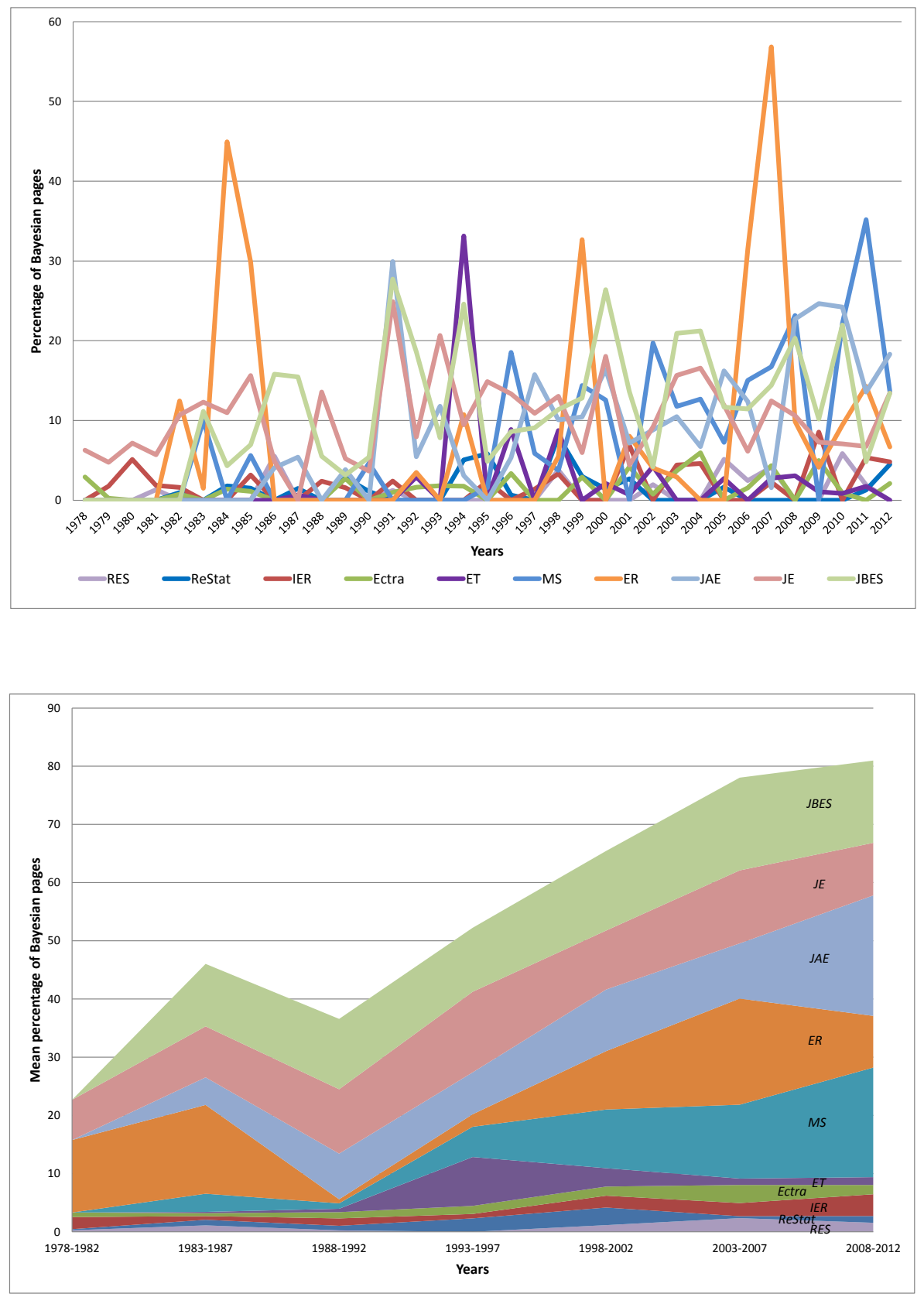

Note: The figures present the annual percentage of pages (top panel) and 5-year averages of pages (bottom panel) of Bayesian papers for the period between 1978 and 2012. In the bottom panel, the final period consists of 4 years. Abbreviations of journals are as follows: Econometrica (Ectra), Econometric Reviews (ER), Econometric Theory (ET), International Economic Review (IER), Journal of Applied Econometrics (JAE), Journal of Business and Economic Statistics (JBES), Journal of Econometrics (JE), Marketing Science (MS), Review of Economic Studies (RES) and Review of Economics and Statistics (ReStat). 


\subsection{Citation Patterns and Impact}

We next focus on citation patterns of papers in the ten journals, as an additional criterion to define the advances in Bayesian econometrics after the 1970s. ${ }^{2}$ The top and bottom left panels in Figure 4 present the number of citations for papers in leading journals during the period between 1978 and 2012. The top and bottom right panels in the figure show the number of citations for a subset of these papers, with at least 400 citations over the sample period. We note that three papers are highly influential in the field with more than 1000 citations: Geweke (1989) (Ectra) with 1228 citations, Kim et al. (1998) (RES) with 1345 citations and Jacquier et al. (1994) (JBES) with 1292 citations. These papers refer to computational advances and financial econometrics with a focus on time varying volatility.

The top panel of Figure 3 shows that the impact analysis is substantially different when we compare the citation pattern with the number of Bayesian pages. Although the journals with a high share of Bayesian pages, ER, JAE, JBES, JE and MS, also have a high share in the total number of citations, there are two high quality theoretical journals, Ectra and RES, with a high influence in the field in terms of the citation numbers despite their relatively low numbers of Bayesian pages. This high impact is more visible when we focus on papers with more than 400 citations, shown in the figures on the right panel of Figure 3. There are 28 papers satisfying this criteria. Note that ER has a large share in total citations although its share in terms of the percentage of pages is more time varying.

The bottom left and bottom right panels in Figure 3 show average citations of papers over 5 year intervals for all cited papers in the journal list and for papers with at least 400 citations, respectively. We first note that the reported average number of citations are naturally low at the end of the sample period, especially between 2008-2012, since these papers are relatively new. When these recent papers are not taken into account, an increasing pattern in the overall number of citations for Bayesian papers is visible: The total numbers presented in the bottom panel of Figure 3 clearly increase between 1978 and 2002. These figures also show that Marketing Science papers started to be cited much more heavily after 1996 and in general the 1990s bring more citations to each journal in our data set.

In order to compare the influential papers in terms of their shares of pages and in terms of the number of citations, we next consider two clusters of journals according to publication patterns in section 3.1 and report the number of citations separately for the journals in these clusters. Figure 4 presents the

\footnotetext{
${ }^{2}$ The citation numbers are collected in the week of 20 August, 2013 for the papers published from 1982 onwards and on 24 October, 2013 for the papers published between 1978 and 1982. The number of citations are based on Google Scholar records available at http://scholar.google.com/.
} 


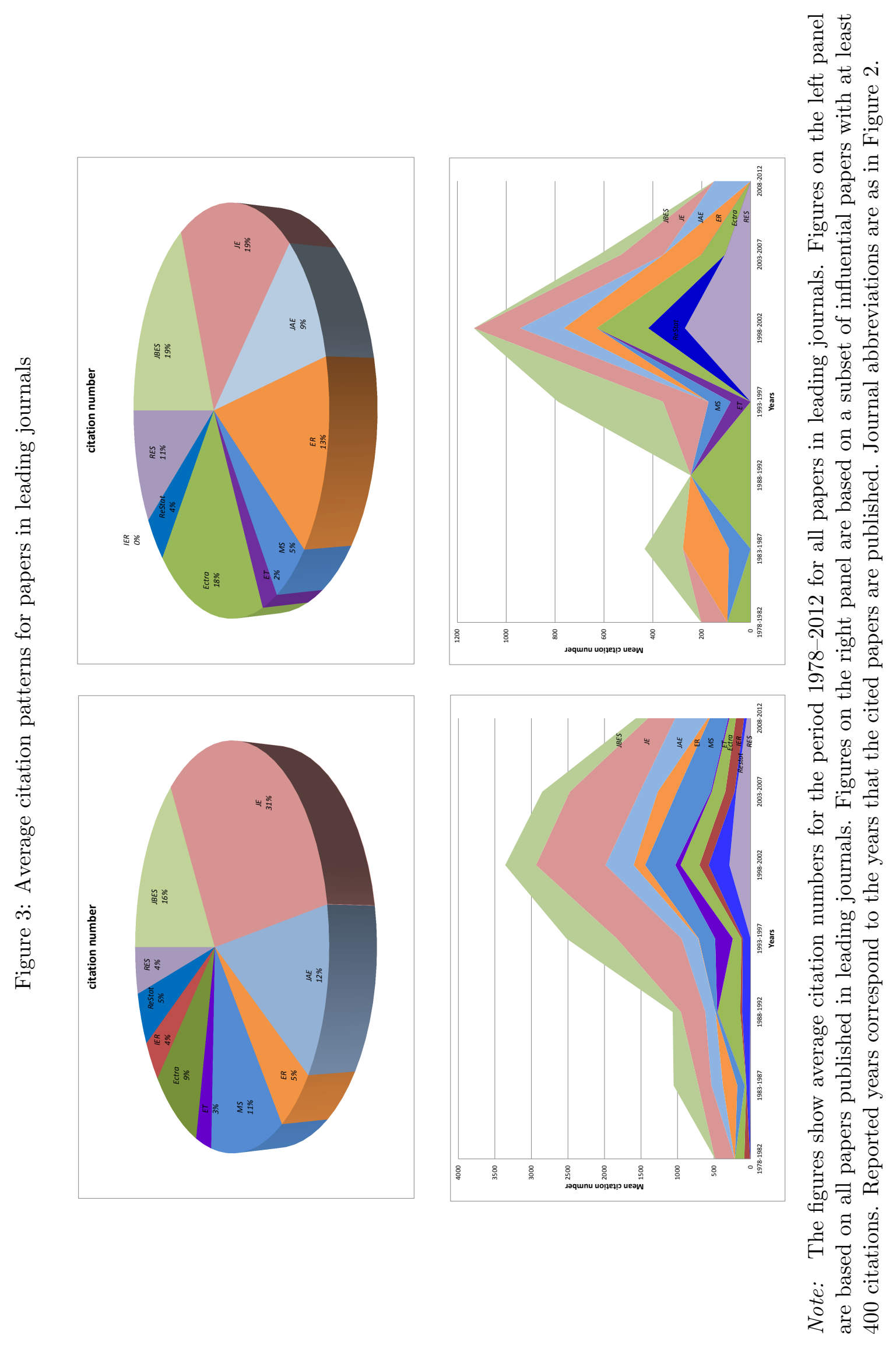


total number of citations for all papers in leading journals and for a subset of influential papers with at least 400 citations for journals classified in cluster 1 and cluster 2. Cluster 1 consists of journals with a relatively low average number of Bayesian pages: Ectra, ET, IER, ReStat and RES. Cluster 2, on the other hand, consists of journals with a relatively high average number of Bayesian pages: ER, JE, JBES, JAE and MS.

The left panel in Figure 4 shows that the journal classification in terms of the percentage of pages is informative about their influence in terms of citations. papers in cluster 2 , journals with a high number of pages devoted to Bayesian econometrics, are on average cited more than those in cluster 1. Despite this similarity, the right panel in Figure 4 shows that highly influential papers with at least 400 citations are more evenly distributed across cluster 1 and cluster 2 journals. Particularly, Ectra and RES have papers that are highly cited.

We conclude that journals which contain both theoretical and applied papers, such as JAE, JE and JBES, publish the large majority of high quality Bayesian econometric papers. Theoretical journals, such as Ectra and RES, on the other hand, publish papers which are highly influential and have a substantial impact on Bayesian research, although the number of these papers are relatively small. Special issues of journals like Econometric Reviews and Econometric Theory receive more citations than usual issues.

\section{Subject Connectivity}

This section considers connectivity of subjects in Bayesian econometric papers. The list of scientific papers in Bayesian econometrics is extensive. We first consider a large set of papers relying on digital archives in order to analyze subject connectivity. We use a random sample of 1000 papers and key terms extracted from each paper provided by the JSTOR digital archive in the field of Bayesian econometrics. ${ }^{3}$ We next refine this extensive list of papers by focusing on the influential ones and summarize the connectivity of key subjects based on this refined set. For each set, the proximities are defined by the number of times that keywords or key terms appear together and in relation to their pairwise concurrence. $^{4}$

We consider two sets of influential papers in the field of Bayesian econometrics. The first set is

\footnotetext{
${ }^{3}$ http://www.jstor.org/

${ }^{4}$ The network maps we present are obtained from the VOSviewer program, available at http://www.vosviewer.com and their software to address proximity, see Waltman et al. (2010); Van Eck and Waltman (2010).
} 

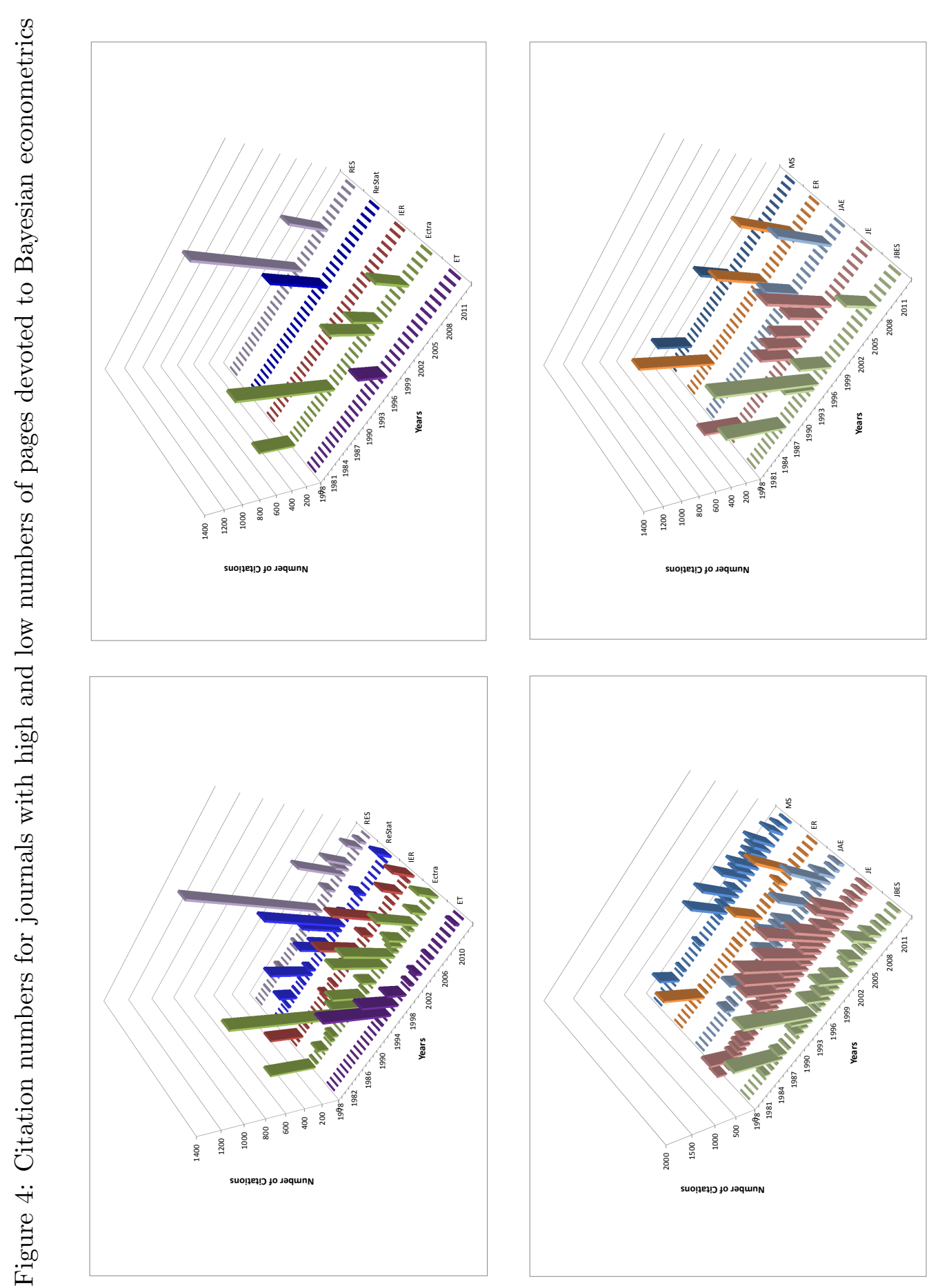

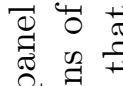

象.

สิ

总要

e

की क्षे

澪

苛范

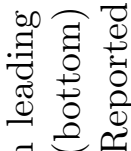

के की मे

究

aे

సี

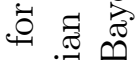

ㄱำ

ণิ ๘

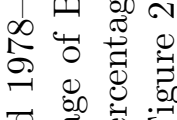

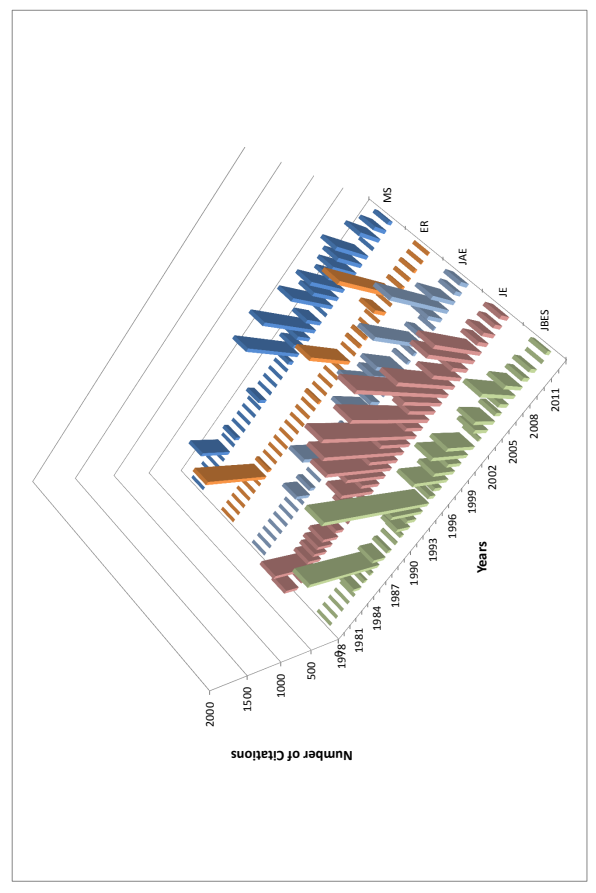

궁

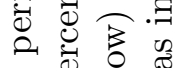

ॠ $\stackrel{0}{2} \stackrel{0}{0}$

운

क व

है

元 $\frac{0}{\sigma}$

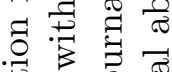

胥 $\frac{\infty}{\sigma} \cdot \frac{0}{\Xi}$

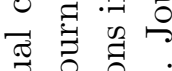

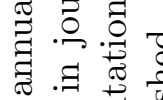

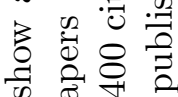

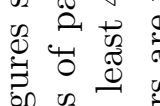

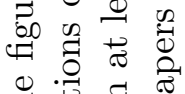

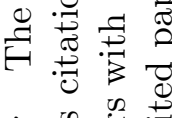

ن.

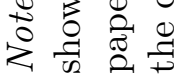


taken from Geweke, Koop, and Van Dijk (2011) and has more than 100 citations according to Google Scholar or Web of Knowledge. ${ }^{5}$ This selection of papers uses expert information, since the set of papers is based on the careful selection of the authors by Geweke, Koop, and Van Dijk (2011). The second set of influential papers are based on the extension of Poirier (1992) presented in section 3.

Figure 5 presents the network and connectivity map for the key terms based on 1000 random papers in Bayesian econometrics, published since 1970. This connectivity analysis is solely based on a random sample of papers that JSTOR provides. Three major areas emerge from this connectivity analysis and these are presented in different colors in Figure 5. The first cluster of keywords, plotted in dark and light green in the figure, corresponds to theoretical topics, with related keywords of likelihood, moment, statistics, assumption and probability. This cluster is naturally linked to all remaining clusters. The second area, consisting of clusters colored in blue and purple, is centered around the key terms 'forecasting' and 'price'. This cluster shows that particularly forecasting is central to the analysis of macroeconomic and financial data, such as (economic) growth, exchange (rates), (financial) returns and interest (rates). Most common models for these data include autoregressive models. (Forecast) horizon, regime (changes) and testing are related and important issues for this area. The third area, shown in light and dark red in Figure 5 has as most prominent key terms 'market', 'choice', 'information' and 'equilibrium'. Other keywords in this area, such as decision, brand, profit, behavior, equilibrium and utility signal market equilibrium models as well as choice models.

The connectivity analysis presented so far does not take into account the amount of influence of each paper, the papers' original keywords or any extra information on the subject area. We next consider a refined set of influential papers in Bayesian econometrics, based on the citations in Geweke, Koop, and Van Dijk (2011). Note that the topics and the references covered in Geweke, Koop, and Van Dijk (2011) are divided to 9 chapters according to the subfields: endogeneity \& treatment (Chapter 1), heterogeneity (Chapter 2), unobserved components \& time series (Chapter 3), flexible structures (Chapter 4), computational advances (Chapter 5), micro \& panel data (Chapter 6), macro \& international economics (Chapter 7), marketing (Chapter 8) and finance (Chapter 9). We consider keywords of Bayesian papers cited in each chapter, and include the corresponding subfield as an additional keyword for each paper.

Figure 6 shows that the subfields defined in Geweke, Koop, and Van Dijk (2011) are connected to

${ }^{5}$ http://scholar.google.com/, http://thomsonreuters.com/web-of-science/. 


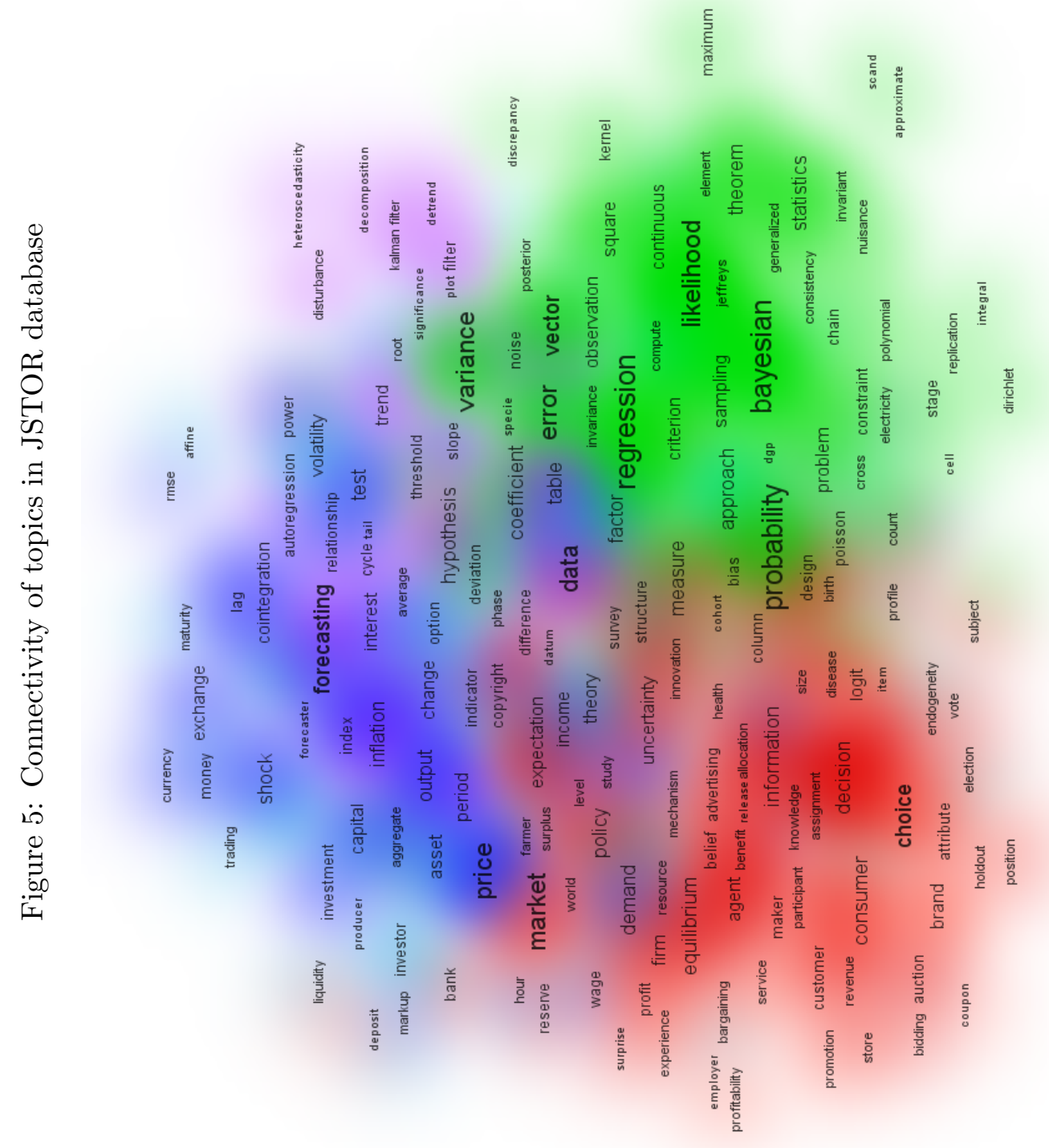

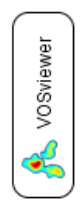




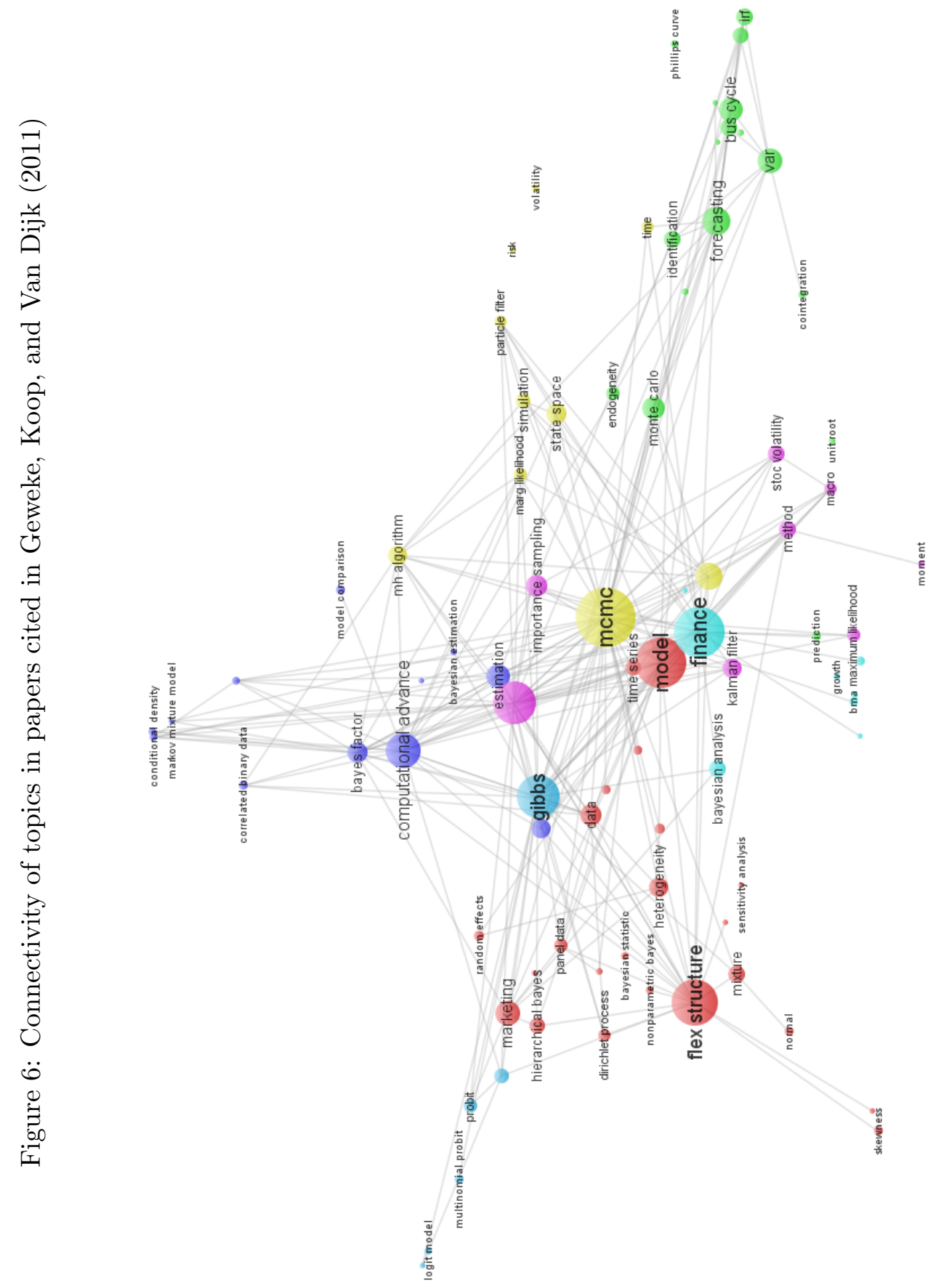

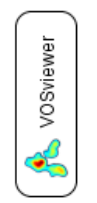


several keywords. This is an expected outcome since we use the chapter information in Geweke, Koop, and Van Dijk (2011) as 'expert knowledge' to relate each paper to a subfield. Besides these subfields, sampling techniques such as the Gibbs sampler, Markov Chain Monte Carlo (MCMC), Metropolis Hastings $(\mathrm{MH})$ algorithm and importance sampling have very large weights indicated by the sizes of the circles in Figure 6 and they lie in the middle of the keyword connection map. This indicates that sampling algorithms are central to research in all subfields of Bayesian econometrics covered here.

An interesting result from Figure 6 is the connectivity of Bayesian methods and economic subfields. Papers in the area of marketing are closely related to flexible model structures (flexible functional forms), and particularly hierarchical Bayes, Dirichlet processes, panel data methods and heterogeneity. Given the increased amount of consumer data in the marketing field, more complex model structures which can handle heterogeneity across consumers are becoming important for this field.

Figure 6 also indicates a strong relation between the macroeconomics and finance literature and Bayesian methods. First, the topic of forecasting is central for macroeconomics and finance as this keyword occurs very frequently and is linked to both areas. Second, state space models, particle filters, Monte Carlo methods, Kalman filter, predictive likelihood analysis and Bayesian Model Averaging (BMA) are closely related to the macroeconomics and finance literature. These close relations indicate the need for sophisticated simulation techniques, such as particle filters, for the estimation and forecasting of complex models used for financial and macroeconomic data. Furthermore, the issue of (parameter) identification is central for macro models used for policy analysis, such as the VAR, Impulse Response Functions (IRF), and business cycle models. This relation is shown in the lower right corner of Figure 6 .

We finally note that computational advances have a large weight according to Figure 6 . This topic is naturally linked to simulation methods, as speeding up computations is a central topic for the wide applicability of simulation methods. Computational advances are central especially for finite mixture models, and are in close relation to the areas of marketing and macro models.

We next select the influential papers in Bayesian econometrics based on the highly (more than 100 times) cited papers published in leading journals in section 3, and analyze the connectivity of the authors and the keywords of each paper. The connectivity of keywords and authors of these papers are shown in Figure 7 using a heatmap of the terms' density estimated by the concurrence of each keyword and author. We note that we leave some authors, such as Atkinson, Dorfman, Gelfand, Griffith and Trivedi, outside Figure 7 for visualization purposes. Despite a high number of papers by these authors, our 
clustering method separates these authors from the central part of the heatmap, most probably due to the diversity of the keywords in these authors' papers.

According to Figure 7, MCMC is central for Bayesian inference and Bayesian analysis. Macroeconomic and finance topics, such as stochastic volatility, time series, DSGE and option pricing, occur frequently in Bayesian econometrics. Marketing and choice models also occur frequently since the second dense area in the heatmap is centered around keywords such as pricing, choice model and advertising.

\section{Topics, Issues and Debates}

In this section we distill topics from section 4 where Bayesian econometrics has shown tremendous progress by itself and also compare it to the frequentist approach. We continue with a set of issues where Bayesian and Non-Bayesian econometricians disagree on and finally we list two debates that Bayesian econometricians hold amongst themselves.

\subsection{Topics}

In this subsection we summarize the advances in five topics which are shown to be central to Bayesian econometrics in section 4 .

\section{The Computational Revolution}

Applicability of Bayesian methods in econometric analysis relies heavily on the feasibility of the estimation of models. For most econometric models of interest the posterior distribution is not of a known form like the normal one and analyzing this distribution and its corresponding model probability using analytical methods or numerical integration methods is infeasible. Monte Carlo (MC) methods have been very useful for tackling these problems. One may characterize this as a 'Computational Revolution' for Bayesian inference leading to statements like 'Monte Carlo saved Bayes'. The popular Markov chain Monte Carlo method, known as Gibbs sampling, contributed in particular to this. Therefore 'Gibbs saved Bayes' is a more appropriate statement.

There are at least three features of MC simulation techniques that make it attractive for Bayesian inference. First, there exists the traditional one of being able to directly simulate a nonlinear function of parameters of a model. Obvious examples are: Given a set of generated draws from parameters of a 


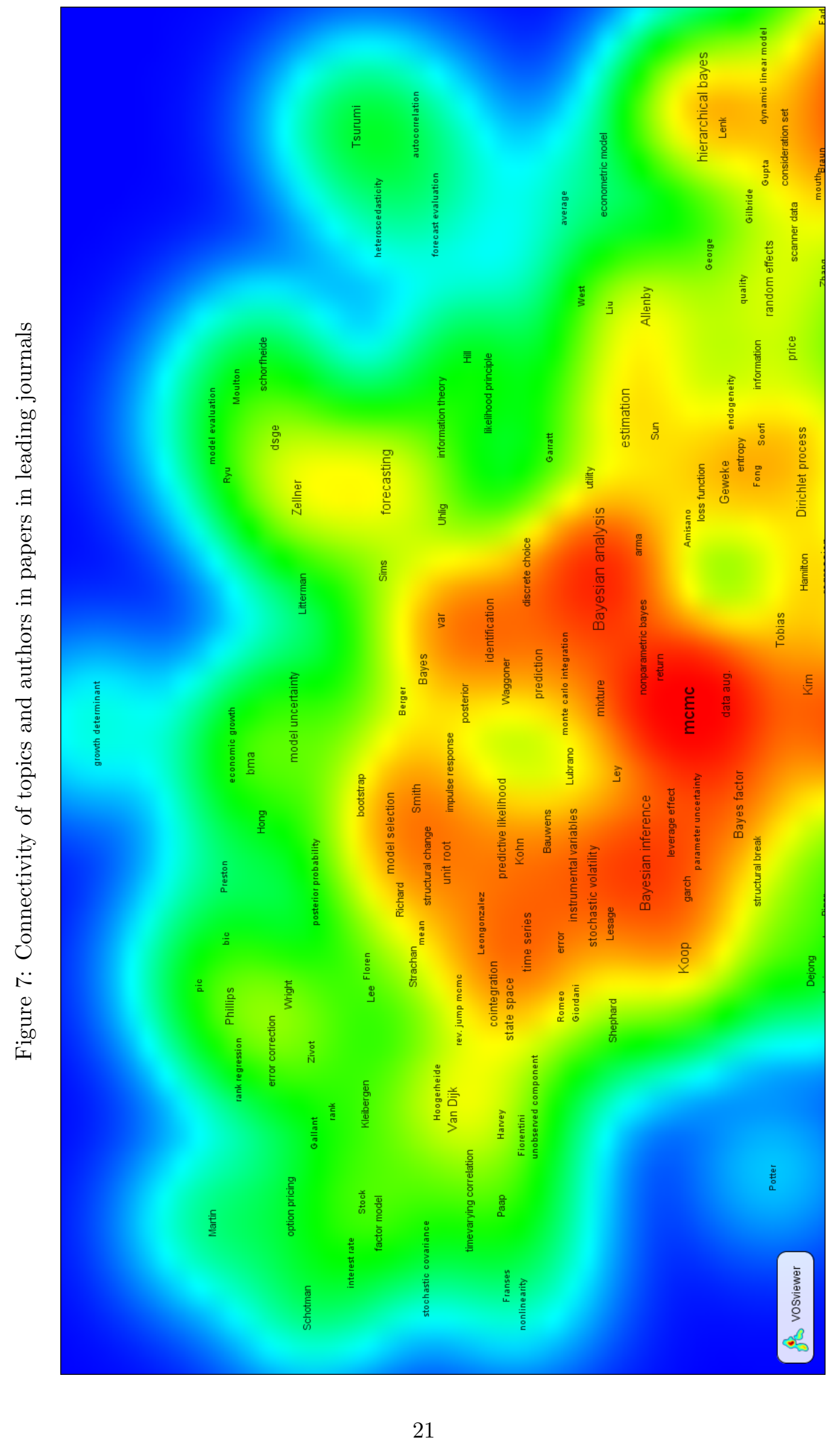




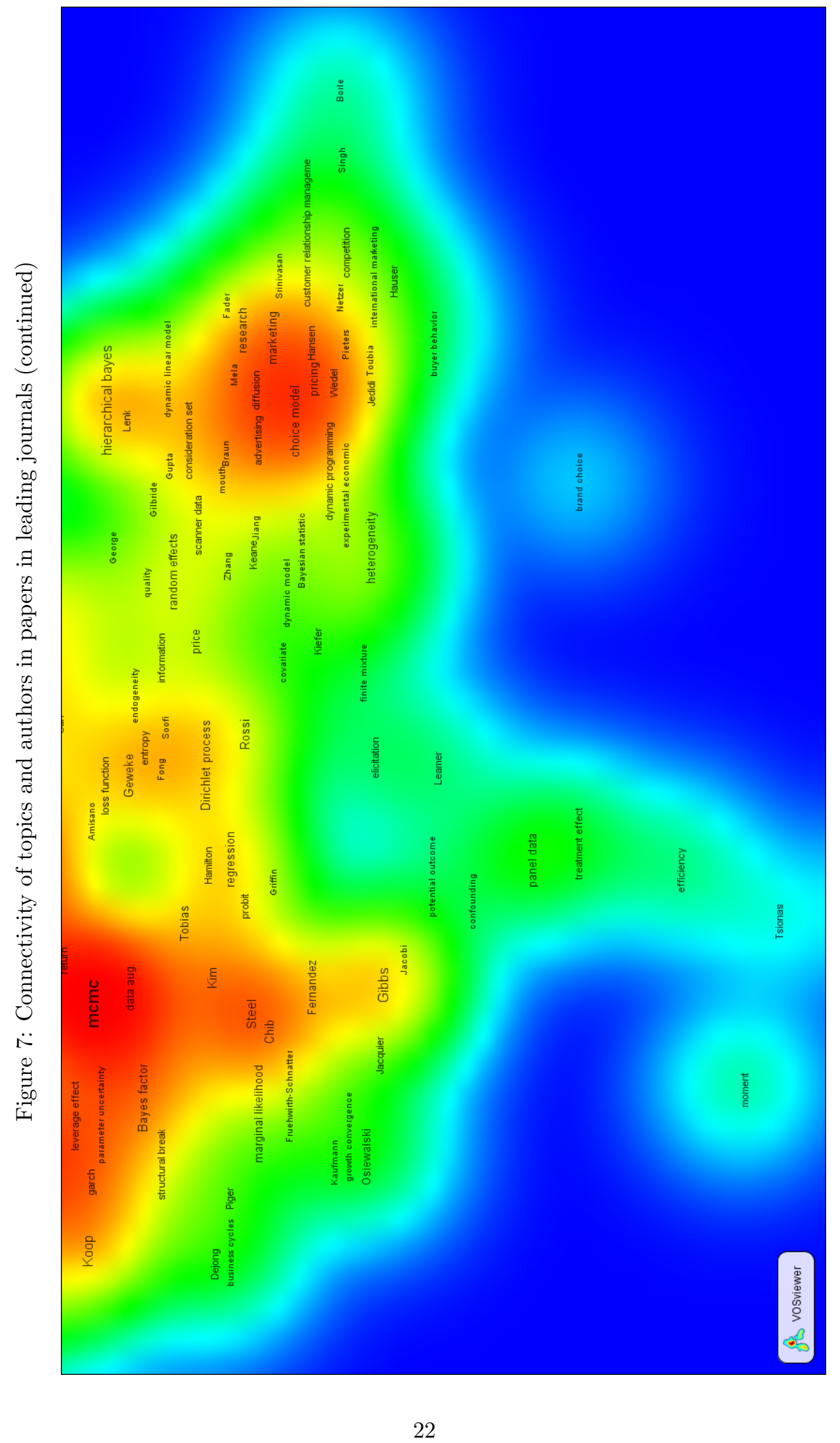


structural model one can directly evaluate the distribution of the multiplier to study the uncertainty of policy effectiveness; and given a set of generated draws from the parameters of a dynamic model one can obtain the distribution of the eigenvalues to study the stability of that system and the random walk nature of the process. These are examples of direct simulation of a model feature given appropriate parameter draws from a model. A second, more methodological feature is how to obtain these parameter draws from models where the posterior is not of a known form and it is not known how to generate draws directly from the posterior. Research in this topic focuses on indirect sampling methods using approximations to the posterior density labeled as importance densities or candidate densities. At first, Importance Sampling (IS), see Hammersley and Handscomb (1964), was introduced in Bayesian inference by Kloek and Van Dijk (1975) and published in Kloek and Van Dijk (1978), further developed by Van Dijk and Kloek $(1980,1985)$ and given a complete detailed treatment in Geweke (1989). The construction of an approximately correct importance function in high dimensions is not trivial and given that the theory of Markov chain Monte Carlo (MCMC) was developed by Metropolis et al. (1953) and Hastings (1970), and extended in several influential papers such as Tierney (1994), this simulation method became the popular one. A major pioneering advance in this first computational revolution is Gibbs sampling developed in Geman and Geman (1984) and extended in Tanner and Wong (1987) and Gelfand and Smith (1990). See Robert and Casella (2004) for a recent and detailed discussion on the Gibbs sampling method and its extensions. The use of sampling methods turned out to be crucial for a third feature of Monte Carlo. Limited dependent variable models including Probit and Tobit models and unobserved component models, in particular State Space models using the Kalman filter, became popular due to their added flexibility in describing nonlinear data patterns. However, these models have an integral in the likelihood that refers to the underlying unobserved continuous data for the limited dependent variable models and unobserved state for the state space models. Bayesian simulation methods that were already used for integration in the parameter space can easily be extended and are the natural technical tools to also integrate these unobserved data and states.

These three features of Monte Carlo contributed greatly to the development of Bayesian econometrics, however, Monte Carlo became operational only with the improvements in the hardware of computing power, i.e. how fast a computer can perform an operation. The issue of computing power is central in econometric analysis in general, but it is even more central to Bayesian econometrics when the MC methods are applied. The improvements in computing power since the 1970s are clearly not negligible, 
but a more recent improvement has been observed with the introduction of clusters of computers, super computers and the possibility of performing operations in Graphical Processing Units (GPUs). These computing power improvements have been immediately adopted in the Bayesian econometrics literature. Using such computing power efficiently, however, mostly requires a careful engineering or modifications in the posterior sampler. Certain sampling methods, such as the importance sampler, are naturally suited for efficient use of computational power, see Cappé et al. (2008) for a discussion. A recent study specifically focusing on enabling Bayesian estimation using the GPU is Durham and Geweke (2013).

\section{Flexible structures, unobserved components models and data augmentation in macroeco- nomics and finance}

Unobserved component models constitute a field in econometrics where Bayesian inference is heavily used. As an example we focus on the state space models in time series analysis. The reason for the extensive use of Bayesian methods in this context is that simulation based Bayesian inference allows for much flexibility in the model structure as well as in the distributional assumptions. Flexible nonlinear structures can be modeled by introducing an extra latent space in such a way that the conditional structure of the model is linear given this unobserved state, see the local level model from Harvey (1990). Then from an estimation point of view, since the unobserved patterns underlying the behavior of observables need to be integrated out of the model, Bayesian integration methods can be used for inference and are very suitable for this class of models. That is, from the inference point of view, Bayesian inference takes the uncertainty of the unobserved patterns into account while estimating the model parameters. This is an important issue where the frequentist approach is more restrictive since the unobserved patterns are estimated conditional on the estimates of the model parameters (one takes the mode of the distribution rather than the whole distribution). Carlin et al. (1992) provide an exposition of the estimation methodology based on simulation to estimate the unobserved components and the model parameters jointly. Shortly after, Jacquier et al. (1994) show how an exact inference, unlike the quasi maximum likelihood approximation, can be obtained for the stochastic volatility models, a popular class of models in finance for modeling time varying volatility, using a similar approach. While the basic Bayesian inference principle remains unchanged, more efficient simulation algorithms are proposed in Carter and Kohn (1994), Fruehwirth-Schnatter (1994), Carter and Kohn (1996), De Jong and Shephard (1995) and Koopman and Durbin (2000). 
Although standard models using unobserved components allow for only continuous changes, models with discrete changes in parameters allowing for structural changes or discrete Markov processes are also feasible using Bayesian techniques. Gerlach and Kohn (2000) and Giordani and Kohn (2008), among others, provide efficient algorithms for obtaining Bayesian inference in case of such discrete changes in parameters. Interesting applications on regime analysis in economics are provided by Paap and Van Dijk (1998) and Sims and Zha (2006).

When the observed variables to be modeled using unobserved components do not follow the standard normal distribution or dependence structures in the model are not linear, other estimation strategies, denoted as Particle Filter or Sequential Monte Carlo techniques that approximate the target distribution to be estimated well, can be conducted. Bayesian inference backed up with advanced simulation algorithms have proved to be very useful in these circumstances, see for example Gordon et al. (1993), Pitt and Shephard (1999), Andrieu and Doucet (2002) and Andrieu et al. (2010). This type of inference is also the key ingredient of the volatility modeling in finance and micro founded macroeconomic models, among others, if the researcher does not resort to linear approximations to estimate the model. This makes it feasible to obtain exact online inference in these settings providing more accurate outcomes. Omori et al. (2007), Fernandez-Villaverde and Rubio-Ramirez (2007) and Fernandez-Villaverde and Rubio-Ramirez (2008) are some examples of this approach.

\section{Choice models, robustness and policy effectiveness}

Hierarchical models that refer to choice processes are a prominent research topic in recent decades due to the, often, unobserved heterogeneity of individual agents in commodity and labor markets. Flexibility in structure and distribution like the Dirichlet process are important features of the modeling process. Latent processes such as Probit models are used to describe unobserved components in models. Panel data are more and more used with scanner data giving rise to massive computing. Basic papers that deal with these issues are McCulloch and Tsay (1994); Rossi et al. (1996) and Hansen et al. (2006). More references are given in Geweke et al. (2011) chapter 8.

Econometric issues in this area are the presence of endogenous regressors, treatment effect problems, latent variables and many parameters. Shrinkage priors are regularly used in this class of models. Gibbsbased MCMC sampling are standard but new simulation based Bayesian techniques are developed using Dirichlet processes in order to gain robustness of results. It is expected that parallel processing will 
become important in this area of Bayesian research.

\section{Instrumental variables}

One of the prominent issues in econometric analysis is endogeneity arising from the correlation between a right hand side variable in an equation and the disturbance of that equation, the so-called direct feedback mechanism. A conventional solution to this problem is the use of instrumental variables (IV), equivalently IV regression models, see e.g. Sargan (1958), Goldberger (1972), and Bowden and Turkington (1990) for a detailed review.

Instrumental Variable (IV) regression model is a single equation Simultaneous Equations Model (SEM). SEMs, incorporating possibly complex feedback mechanisms between series, have been analyzed in the Cowles Commission monographs (Koopmans, 1950; Hood and Koopmans, 1953) and have been widely employed to analyze the behavior of markets, macroeconomic and other multivariate systems.

Bayesian analysis has been popular in these class of models due to the parameter identification issues plaguing inference in these models, in line with identification issues in SEMs in general. Bayesian analysis of the IV regression models are introduced by Lindley and El-Sayyad (1968), Drèze (1976) and Drèze (1977). Earlier literature suggests that the posterior densities in these class of models under flat priors may be improper, see e.g. Zellner, Bauwens, and Van Dijk (1988) and Bauwens and Van Dijk (1990). An exception of this result is the case of over-identification where the posterior densities are shown to be proper under flat priors as shown in Zellner, Ando, Baştürk, Hoogerheide, and Van Dijk (2014).

Due to the identification issues in IV regression models, the use of alternative prior structures, such as the Jeffrey's prior, are proposed in Kleibergen and Van Dijk (1998) and Hoogerheide, Kaashoek, and Van Dijk (2007). More recent advances in the Bayesian estimation of these models are the introduction of semiparametric models by Conley et al. (2008) and Florens and Simoni (2012) among others, and efficient posterior sampling algorithms as in Zellner, Ando, Baştürk, Hoogerheide, and Van Dijk (2014). For a detailed discussion of Bayesian approaches to IV and many examples, we refer to Lancaster (2004) and Rossi et al. (2005).

\section{Dynamic models and forecasting}

Bayesian analysis has become a dominant forecasting and counterfactual analysis tool in recent decades. There are four main reasons for this phenomenon. First, many of the complex, otherwise non-estimable, 
models can be estimated using simulation based Bayesian methodology. Perhaps, the most important example of these models includes the class of structural micro founded macroeconomic models, such as Dynamic Stochastic General Equilibrium models, that are used both for policy analysis and for forecasting, see for example Smets and Wouters (2003), Smets and Wouters (2007), An and Schorfheide (2007). Currently, many of the central banks employ such models to obtain short and long term projections of the economy. An advantage of the Bayesian methodology is that it provides a solid statistical ground for efficient analysis using these structural models. As Bayesian inference provides the distribution of many key parameters that play a crucial role in economic analysis it is often used as a tool for counterfactual analysis. For instance, the questions such as 'if quantitative easing were not conducted in US, would the course of the recession differ?' could be answered by estimating relevant structural models. Bayesian analysis provides a statistically coherent tool for employing counterfactual analysis by forecasting under counterfactuals.

Second, prior distributions can play an integral part of the forecasting especially for the overparametrized models. Vector Auto Regression models (VAR) are major examples where Bayesian inference facilitates forecasting using the prior distributions for shrinking the parameters towards zero and thereby decreasing the dimensionality of the models. Decreasing the dimension of the overparametrized models using clever prior distributions has proved to be very useful in many applications. Prominent examples of this approach constitute Doan et al. (1984), Kadiyala and Karlsson (1997), Banbura et al. (2010).

Third, Bayesian methodology takes the parameter uncertainty into account which may be of crucial importance in many applications. This enables researchers to obtain the entire predictive distribution rather than point forecasts based on the mode of the parameter distribution as in the frequentist approach. An important advantage of this feature is that different parts of the predictive distribution can be analyzed easily. This yields an obvious advantage for the analysis of various types of risk in finance and macroeconomics. A recent example is given in Basturk, Cakmakli, Ceyhan, and Van Dijk (2013a) where the probability of deflation is evaluated for the US.

Fourth, the Bayesian methodology provides a natural and statistically solid way to take model uncertainty into account and to combine models to increase the predictive ability of many competing models. Bayesian model averaging technique provides one elegant way to do so, see for example Min and Zellner (1993), Fernandez et al. (2001), Avramov (2002), Cremers (2002) and Wright (2008). Recent advances 
in Bayesian model combination also allows to combine models where the model space is not complete implying that none of the competing models might be the true model. In that case, optimal combinations are proposed in Geweke and Amisano (2010) and Geweke and Amisano (2011). For a recent example where Sequential Monte Carlo is used to obtain density combinations from different model structures we refer to Billio, Casarin, Ravazzolo, and van Dijk (2013).

\subsection{Issues}

We next summarize three issues in Bayesian econometrics that non-Bayesian repeatedly referred to in the literature, and we discuss the advances in Bayesian econometrics regarding these issues.

\section{Identification, the value of prior information and model evaluation}

The choice of a reasonable informative prior distribution is a crucial part of the Bayesian analysis and often subject to criticism by frequentist econometricians. However, sensible prior distributions provides valuable improvements in inference for many of the econometric issues that are hard to tackle without it. Hence, it is a bless rather than a curse. Prior distributions play a key role in many aspects such as identification and incorporation of prior knowledge or evidence from other studies, dimension reduction and forecasting, and nonparametric Bayesian inference.

In many cases, data are not sufficiently informative about the appropriate parameter values and may yield similar likelihood values with different parameter combinations, which is referred to as 'the weak identification problem'. Weak identification occurs in models with nearly reduced rank, which occurs in simultaneous equations models, instrumental variable regression models, dynamic models with cointegration and in factor models. Weak identification gives usually irregular behavior of the likelihood; see papers by Bauwens and Van Dijk (1990), Kleibergen and Van Dijk (1994), Kleibergen and Van Dijk (1998) and Hoogerheide, Kaashoek, and Van Dijk (2007). In such cases, assigning reasonable priors from other studies or other evidence alleviate the identification problem since Bayesian inference incorporates prior knowledge with the data information using the Bayes rule. This is often used in micro founded macroeconomic studies where priors are constructed using economic theory or from other studies or from micro data such as households surveys, see for example Del Negro and Schorfheide (2008). Another well known example of prior information is the use of reasonable regions in the parameter space, restricted by inequality conditions. Frequentist inference is extremely difficult using such restrictions. Examples 
of Bayesian inference where the implied prior on the range of an economics multiplier or a prior on the length of the period of oscillation of the business cycle yield plausible restrictions are given in Van Dijk and Kloek (1980) and Harvey, Trimbur, and Van Dijk (2007).

One important issue is the existence of the posterior distribution and its moments in case of weak or noninformative priors and an almost flat likelihood. Many Bayesians argue that the posterior distribution does not exist in such a case. However, Zellner, Ando, Baştürk, Hoogerheide, and Van Dijk (2014) shows that this may not be correct and give very weak conditions for existence of moments of zero and higher order for the famous weak instrument problem. Priors play an instrumental role in forecasting especially when the models are overparametrized. Often overparametrized models are condemned with poor forecasting performance as any additional parameters exploits the data information further and yielding prediction uncertainty. To tackle this problem sensible prior distributions are assigned on parameters shrinking those parameters that are not informative towards zero and thereby decreasing the dimension of the problem. In macroeconomic forecasting the priors proposed by Doan et al. (1984) have become a standard tool among econometricians in academia and in other institutions such as central banks. In more general cases, many tailored priors are used for shrinkage of the model parameters towards zero and therefore they are efficiently used in variable selection when there are many candidate variables to select from. Prominent examples include, George and McCulloch (1993), Ishwaran and Rao (2005) and Park and Casella (2008) among others.

Prior distributions are also the key ingredients for flexible modeling strategies in Bayesian analysis. This is especially of key importance for density estimation. Bayesian nonparametric analysis is one evolving area where such prior distributions or processes are heavily used. While some of the theoretical achievements were already accomplished during 1970s, see Ferguson (1973), Antoniak (1974) for example and Sethuraman (1994) for a more recent paper, extensive use of such priors were only possible with the advance of computing power. Indeed with increasing computing power simulation schemes used for Bayesian nonparametric inference proved to be very useful for such complex analysis, see for example Escobar and West (1995), Neal (2000) and Walker (2007). Currently, many applications have emerged in different fields using such flexible prior distributions, see for example Chib and Hamilton (2002); Hirano (2002); Griffin and Steel (2004); Jensen (2004); Jensen and Maheu (2010).

Lindley's paradox - or Bartlett's or Jeffreys' paradox; see Lindley (1957) and Bartlett (1957) - implies that one has to choose very carefully the amount of prior information compared to the amount of sample 
information, when comparing alternative hypotheses on model structures with the intention to let the information from the data in the likelihood dominate that of the prior. Typically a naive or malevolent researcher could 'force' the posterior probability of a certain model M, the 'restricted model' in case of two nested models, to tend to go to unity by letting the priors in all alternative models tend to diffuse priors, thereby decreasing the marginal likelihoods of all alternative models, even if the particular model $\mathrm{M}$ does not make sense and poorly describes the data. In an attempt to make the posterior model probabilities 'fair', one could use predictive likelihoods instead of marginal likelihoods; see Gelfand and Dey (1994), O'Hagan (1995), and Berger and Pericchi (1996). However, the use of predictive likelihoods brings several questions and issues. First, one must choose the training sample and the hold-out sample. Examples of important questions are: How many observations are included in these samples? Is one training sample used or does one average over multiple (or all possible) training samples? In the latter case, what does one average e.g., marginal likelihoods, logarithms of marginal likelihoods, Savage-Dickey Density Ratios or posterior model probabilities? Second, if one chooses to average results over multiple (or all possible) training samples, then the computing time that is required for obtaining all Monte Carlo simulation results for all training samples may be huge. In other words, the Lindley paradox and the computation of predictive likelihoods enlarge the relevance of simulation methods that efficiently provide reliable and accurate results in case of non-elliptical credible sets. A suitable method must deal with large numbers of different non-elliptical shapes in a feasible computing time. For time series models computing the marginal likelihood for a random subsample implies that the estimation must be performed for an irregularly observed time series (with many 'missing values'), which is typically only feasible using an appropriate formulation and estimation of a state space model. In future research computationally efficient and accurate simulation methods need to be developed here. ${ }^{6}$

\section{Dynamic inference and nonstationarity}

Dynamic inference and methods to handle nonstationary data constitute a second Bayesian topic subject to criticism by frequentist econometricians. As a start we summarize the perfect duality between Bayesian inference in the parameter space and frequentist inference in the sample space for the well-known class of the linear regression model $y=X \beta+\epsilon$. In both frequentist and Bayesian econometrics, the parameter $\beta$ has a student- $t$ density. However, the interpretations are different. A graphical illustration of the

\footnotetext{
${ }^{6}$ Part of this paragraph is taken from Van Dijk (2013).
} 
Table 1: Duality and differences between Bayesian and frequentist inference

\begin{tabular}{|l|l|}
\hline Classical inference & Bayesian inference \\
\hline Parameters $\beta$ are fixed unknown constants & $\begin{array}{l}\text { Parameters } \beta \text { are stochastic variables. One de- } \\
\text { fines a prior distribution on parameter space. }\end{array}$ \\
\hline $\begin{array}{l}\text { Data } y \text { are used to estimate } \beta \text { and check va- } \\
\text { lidity of postulated model, by comparing data } \\
\text { with (infinitely large, hypothetical) data set } \\
\text { from model. }\end{array}$ & $\begin{array}{l}\text { Data } y \text { are used as evidence to update state of } \\
\text { the mind: data transform prior into posterior } \\
\text { distribution using the likelihood. }\end{array}$ \\
\hline $\begin{array}{l}\text { Objective concept of probability: Probability } \\
\text { is the fraction of occurrences when the process } \\
\text { is repeated infinitely often. }\end{array}$ & $\begin{array}{l}\text { Subjective concept of probability: Probability } \\
\text { is a degree of belief that an event occurs. }\end{array}$ \\
\hline $\begin{array}{l}\text { One can use the maximum likelihood estimator } \\
\text { as an estimator of } \beta .\end{array}$ & $\begin{array}{l}\text { One uses Bayes' theorem to obtain the poste- } \\
\text { rior distribution of } \beta \text {. One can use } E(\beta \mid y) \text { or } \\
\text { minimize a loss function to estimate } \theta .\end{array}$ \\
\hline $\mathrm{R}^{2}$ is used to compare models. & $\begin{array}{l}\text { Model comparison is carried out by using pos- } \\
\text { terior odds ratio. }\end{array}$ \\
\hline
\end{tabular}

difference for this model is provided in the left panel in Figure 8. Table 1 presents a summary of the duality and differences between Bayesian and frequentist inference. In practice one finds that many empirical researchers are 'closet' Bayesians in interpreting the obtained value of a t-test as indicating possible positive strength of the empirical result. In the strict frequentist sense one can only reject a null hypothesis if there is sufficient evidence for it.

This equivalence breaks for dynamic regression models. In inference about stationary dynamic models, while Bayesian econometrics suggests student- $t$ density for the parameters, the frequentist econometrics has finite sample bias problems. This divergence between the Bayesian and the frequentist inference is also observed for nonstationary dynamic models, see Sims and Uhlig (1991). In the frequentist case, the inferential statement: 'No falsification of the Null Hypothesis of a Unit Root leads to the acceptance of the Unit Root' yields fragile and often incorrect conclusions, for example, if a break occurs in the series. That is, several alternatives may be more plausible.

Sims and Uhlig (1991) and Schotman and Van Dijk (1991b), Schotman and Van Dijk (1991a) suggest that Bayesian inference for models with unit root is more sensible, as well as much easier to handle analytically, than the classical confidence statements. Even under the assumptions of linearity and Gaussian disturbances, and even if conditioning on initial conditions is maintained, classical small-sample distribution theory for autoregressions is complicated. Classical asymptotic theory breaks discontinuously at the boundary of the stationary region. Therefore, the usual simple normal asymptotic approximations 
Figure 8: Frequentist versus Bayesian econometrics

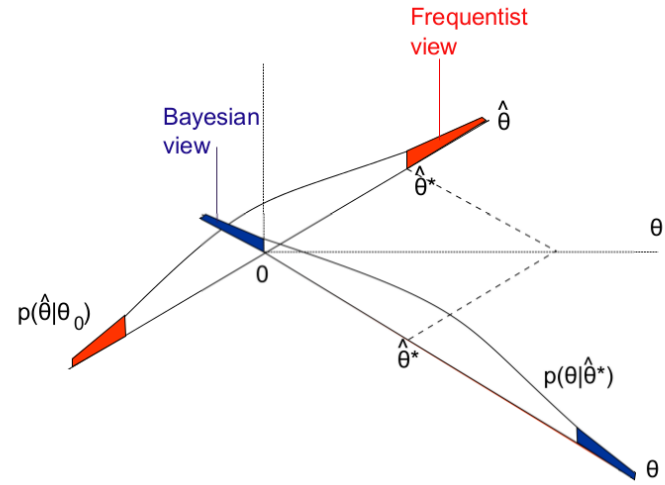

Static inference

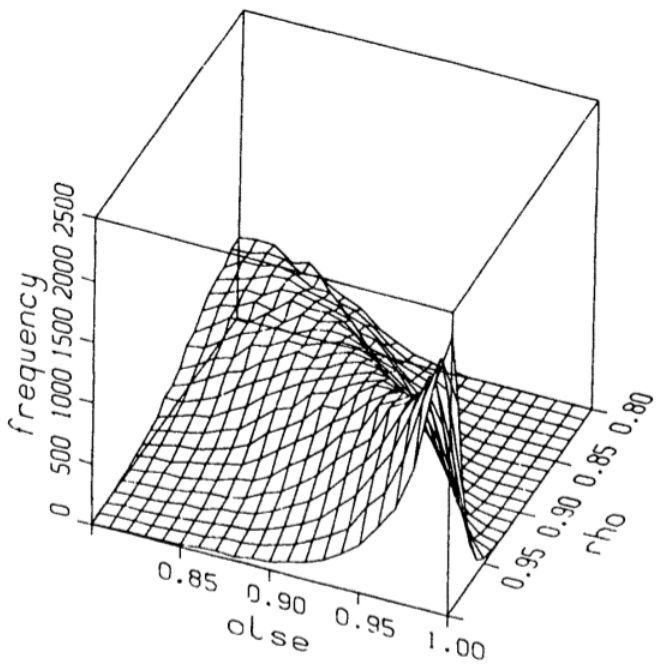

Dynamic inference (Sims and Uhlig, 1991)

are not available. The likelihood function, however, is well known to be the same in autoregressions and non-dynamic regressions, assuming independence of disturbances from lagged dependent variables. Thus inference satisfying the likelihood principle has the same character in autoregressions whether or not the data may be non-stationary. The illustration of the likelihood for this autoregressive model in Sims and Uhlig (1991) is given in the right panel of Figure 8. Phillips (1991) stresses the fragility of Bayesian inference to the specification of the prior and warns against the mechanical use of a flat prior. Schotman and Van Dijk (1991b) present a solution to this problem in a different parameterizations of this model. Schotman and Van Dijk (1991a) suggest to use posterior odds test (for the choice between a unit root model and an $\mathrm{AR}(1)$ stationary model). In the Bayesian approach, a unit root is not a testing problem but a choice problem on the relative weights of two states of nature: the stationary and the nonstationary case, see Sims and Uhlig (1991). One can use these weights in evaluating forecasts and impulse response functions. De Pooter, Ravazzolo, Segers, and Van Dijk (2009) suggest to use the Schotman-van-Dijk (SVD) prior in this context.

The difference between Bayesian and frequentist econometrics is particularly apparent for the case of multivariate dynamic models with possible nonstationarity. Kleibergen and Van Dijk (1993) propose a Bayesian procedure to inference and show that by using flat priors, the marginal posteriors of the cointegration vectors are ill-behaved when certain parameters become non-identified. This problem also plagues standard frequentist inference. Kleibergen and Van Dijk (1993) solve this problem by proposing 
the Jeffrey's prior. The key problem with the frequentist approach is that a sequential testing procedure is used to determine the number of stationary and the number of nonstationary relations, in other words how many stable and unstable relations exist. In the Bayesian approach weights can be evaluated for each member of the set of stable and unstable relations. Forecasts can be made and impulse responses evaluated with a weighted average of such relations using marginal and predictive likelihoods, see Wright (2008) and Strachan and Van Dijk (2013) for details.

\section{Vector autoregressive versus structural modeling}

Given that in the early 1970s oil price shocks and high inflation affected macroeconomic time series both in levels and volatility, the existing classes of econometric models often based on Keynesian structures did not fit and forecast well. In the words of Christopher Sims these models were not 'realistic'. In his 1980 paper in Econometrica, Sims advocated the use of Vector Autoregressive Models (VAR) to describe better the time series patterns in the data. One may characterize his work as: Sims against the 'Econometric Establishment'. However pragmatic the VAR approach was, there quickly were discussions on the fact that the unrestricted VAR had the curse of parameter dimensionality or otherwise stated an over-parametrization danger. Several approaches to overcome this criticism were developed of which we discuss the following ones. One approach is to make use of shrinkage priors that were of great help in forecasting. This class of priors became known as the Minnesota prior from Doan et al. (1984). A useful alternative is the dummy based observation prior due to amongst others Kadiyala and Karlsson (1997)). In the late 1990s structural economic priors Del Negro and Schorfheide (2004) came into existence parallel to the use of more structural VAR models like the DSGE model from (Smets and Wouters, 2007) and many other Structural VAR's. This latter topic has been discussed before. Nowadays structural VARs with informative priors are used everywhere in macroeconomics in academia and professional organizations both for forecasting and policy analysis. Given the recent economic crisis it is clear that this class of models needs to be developed further to include financial sectors.

\subsection{Debates}

This section summarizes a major debate between Bayesian econometricians, namely the choice of objective versus subjective econometrics. We then summarize the attempts to improve communication between statistics and econometrics. 
Table 2: Objective versus subjective econometrics

\begin{tabular}{|l|l|}
\hline Objective & Subjective \\
\hline $\begin{array}{l}\text { Let the model speak: analyze the shape of the } \\
\text { likelihood }\end{array}$ & Everything is personal \\
\hline Scientific evidence dominates & Personal probabilities should be solicited \\
\hline Experts opinion may fail & Experts opinions matter \\
\hline Reach a large public & \\
\hline
\end{tabular}

\section{Objective versus subjective econometrics}

In general, probabilities are not physical quantities like weight, distance, or heat that one can measure using physical instruments. As De Finetti (1989) suggested, 'Probabilities are a state of the mind'. Therefore, in general Bayesians are subjectivists and probabilities are personal statements. However, some are more subjectivist/personal than others.

The alternative, objectivist, viewpoint is based on the idea that experts opinions may fail, and aims to 'Let the model speak' in reporting scientific evidence. This viewpoint, due to limited inclusion of personal or expert statements, reaches a large public. The subjective viewpoint, on the other hand, argues that even when experts opinions fail, the likelihood will show that this prior is not relevant. A brief summary of the differences between the subjectivist and objectivist viewpoints are provided in table 2 .

In conclusion, there exist 'true' Bayesian econometricians who belong in the right hand column of table 2; Instrumental and pragmatic Bayesian econometricians who belong more in the left column of table 2; Pragmatic Bayesian econometricians and 'Closet' Bayesian econometricians those that use regression outcomes and talk about 'strongly significant' $t$-values and 'accept' the null hypothesis. They all apply Bayesian techniques nowadays!

\section{Communication between Statistics and Econometrics}

Statistics and Econometrics has had a difficult relationship, with several switches in the past 50 years, due to the fact that econometric models are high-dimensional while statisticians prefer a maximum of 3 dimensions. Early statistics was applied to economic time series while recent statistics is applied more to biology and is becoming very computational. Econometrics is more model-oriented with a large number of parameters. 
There have been attempts to construct bridges between statistics and econometrics. Among these are the Seminar on Bayesian Inference in Econometrics and Statistics (SBIES) that was pioneered by Arnold Zellner from 1970 onwards and now actively steered by Siddharta Chib ${ }^{7}$, the European Seminar in Bayesian Econometrics (ESOBE) that started in 2010 by Herman K. van Dijk ${ }^{8}$ and the Economics, Finance and Business Section (EFAB) of the International Society of Bayesian Analysis (ISBA) that was started in 2013 by Mike West. ${ }^{9}$

\section{Conclusion}

Inspired by the path that Bayesian econometrics has followed for the last half century, this section presents the authors' personal expectations of the important topics/subjects for the future of Bayesian econometrics in the $21^{\text {st }}$ Century. One such prediction is that 'the second computational simulation revolution' where efficient information distillation from 'big data' with sophisticated Bayesian methodology using parallelization techniques is going to play an important role. Another topic that is predicted to gain popularity is complex economic structures with nonlinearities and complete predictive densities. A third topic that is expected to have importance in the future is the analysis of implied model features, such as risk or instability due to diverging ratios, and decision analysis. Finally, model incompleteness, which refrains from the assumption that the true data generating process is in the defined model set, is predicted to be important topics in Bayesian econometrics, see Geweke (2010).

Besides focusing on important topics in Bayesian econometrics, we further predict that the influence of Bayesian econometrics in the econometrics field will continue to increase over time. This final prediction is in line with the statement 'Econometrics should always and everywhere be Bayesian' in Sims (2007). We refer to Sims (2007) for a detailed discussion on this topic and on how Bayesian approaches might become more prevalent in several areas of economic applications

We end this paper with a bit of a game. The citation numbers we analyze for Bayesian papers can be related to the h-index of authors, a conventional measure for the impact of published work by scholars, see Hirsch (2005). Nowadays, the h-index is sometimes used in the career path and promotion stages of young researchers. We employ a simple simulation study to assess the expected h-index of a 'random

\footnotetext{
7 apps.olin.wustl.edu/conf/sbies/Home/

8 ww. esobe.org/

${ }^{9}$ bayesian.org/sections/EFaB/bylaws
} 
Bayesian' publishing a predefined number of papers in the leading journals we consider. In order to assess this expected h-index, we consider a random sample of size $J$ from the 928 papers in our database and calculate the h-index. The average h-index for 1000 such random samples is used to approximate the expected h-index for an author with $J$ publications in leading journals. For a young Bayesian econometrician who is the author of 5 such publications, we find that the h-index is approximately 4 , i.e. very high compared to the total number of publications of this author, and the expected number of citations for this author's papers is 334. For an author, coming up for tenure, with 12 publications in leading journals the expected h-index is approximately 9 with an expected number of citations of 765 . For a very established author with 50 publications in these journals, the expected h-index is approximately 25, with an expected number of citations of 1644. The papers and the journals considered therefore have a considerable impact in the field, according to the calculated h-indexes. Conditional upon our data set we conclude that young Bayesian econometricians have a very good chance to follow an academic career successfully. 


\section{References}

Aldrich, J. 1995. R. A. Fisher and the making of Maximum Likelihood 1912-22. Discussion Paper Series In Economics And Econometrics 9504, Economics Division, School of Social Sciences, University of Southampton.

An, S. and F. Schorfheide. 2007. Bayesian analysis of DSGE models. Econometric Reviews, 26(2-4): $113-172$.

Anderson, T.W. 1947. A note on a maximum-likelihood estimate. Cowles Foundation paper 21c, Cowles Commission. Reprinted in Econometrica (1947) 15(3): 241-244.

Anderson, T.W. and H. Rubin. 1949. Estimation of the parameters of a single equation in a complete system of stochastic equations. Cowles Foundation paper 36a, Cowles Commission. Reprinted in Annals of Mathematical Statistics (1949) 20(1): 46-63.

Andrieu, C. and A. Doucet. 2002. Particle filtering for partially observed Gaussian state space models. Journal of the Royal Statistical Society: Series B (Statistical Methodology), 64(4): 827-836.

Andrieu, C., A. Doucet, and R. Holenstein. 2010. Particle Markov chain Monte Carlo methods. Journal of the Royal Statistical Society: Series B (Statistical Methodology), 72(3): 269-342.

Antoniak, Charles E. 1974. Mixtures of Dirichlet processes with applications to Bayesian nonparametric problems. The annals of statistics: 1152-1174.

Avramov, Doron. 2002. Stock return predictability and model uncertainty. Journal of Financial Economics, 64(3): 423-458.

Banbura, M., D. Giannone, and L. Reichlin. 2010. Large Bayesian vector auto regressions. Journal of Applied Econometrics, 25(1): 71-92.

Bartlett, M.S. 1957. Comment on 'A statistical paradox' by D.V. Lindley. Biometrika, 44(1-2): 533-534.

Basturk, N., C. Cakmakli, P. Ceyhan, and H.K. Van Dijk. 2013a. Posterior-predictive evidence on US inflation using extended Phillips curve models with non-filtered data. Accepted in Journal of Applied Econometrics.

Basturk, N., L.F. Hoogerheide, and H.K. Van Dijk. 2013b. Measuring returns to education: Bayesian analysis using weak or invalid instrumental variables. Unpublished manuscript.

Bauwens, L. and H.K. Van Dijk. 1990. Bayesian limited information analysis revisited. In J.J. Gabszewicz, J.F. Richard, and L.A. Wolsey (eds.), Economic Decision-Making: Games, Econometrics and Optimisation: Contributions in Honour of Jacques H. Drèze, North Holland, chapter 18. 385-424.

Berger, J.O. and L.R. Pericchi. 1996. The intrinsic Bayes factor for linear models. Bayesian statistics, 5: $25-44$.

Billio, Monica, Roberto Casarin, Francesco Ravazzolo, and Herman K. van Dijk. 2013. Time-varying combinations of predictive densities using nonlinear filtering. Journal of Econometrics, 177(2): 213232 .

Bos, C.S., R.J. Mahieu, and H.K. Van Dijk. 2000. Daily exchange rate behaviour and hedging of currency risk. Journal of Applied Econometrics, 15(6): 671-696. 
Bowden, R.J. and D.A. Turkington. 1990. Instrumental variables. Cambridge University Press.

Brainard, W.C. 1967. Uncertainty and the effectiveness of policy. The American Economic Review, 57(2): pp. 411-425.

Cappé, O., R. Douc, A. Guillin, J.M. Marin, and C.P. Robert. 2008. Adaptive importance sampling in general mixture classes. Statistics and Computing, 18(4): 447-459.

Carlin, B.P., N.G. Polson, and D.S. Stoffer. 1992. A Monte Carlo approach to nonnormal and nonlinear state-space modeling. Journal of the American Statistical Association, 87(418): 493-500.

Carter, C. K. and R. Kohn. 1994. On Gibbs sampling for state space models. Biometrika, 81(3): $541-553$

Carter, C. K. and R. Kohn. 1996. Markov chain Monte Carlo in conditionally Gaussian state space models. Biometrika, 83(3): 589-601.

Chernoff, H. 1954. Rational selection of decision functions. Cowles Foundation paper 91, Cowles Commission. Reprinted in Econometrica (1954), 22: 422-443.

Chernoff, H. and N. Divinsky. 1953. The computation of maximum-likelihood estimates of linear structural equations. Studies in Econometric Method, 14: 236-302.

Chib, S. and B. Hamilton. 2002. Semiparametric Bayes analysis of longitudinal data treatment models. Journal of Econometrics, 110(1): 67-89.

Conley, T.G., C.B. Hansen, R.E. McCulloch, and P.E. Rossi. 2008. A semi-parametric Bayesian approach to the instrumental variable problem. Journal of Econometrics, 144(1): 276-305.

Cremers, K.J.M. 2002. Stock return predictability: A Bayesian model selection perspective. Review of Financial Studies, 15(4): 1223-1249.

De Finetti, B. 1989. Probabilism. Erkenntnis, 31(2): 169-223.

De Jong, P. and N. Shephard. 1995. The simulation smoother for time series models. Biometrika, 82(2): 339-350.

De Pooter, M., F. Ravazzolo, R. Segers, and H.K. Van Dijk. 2009. Bayesian near-boundary analysis in basic macroeconomic time-series models. In S. Chib, G. Koop, W. Griffiths, and D. Terrell (eds.), Advances in Econometrics, (Bayesian econometrics), Emerald Group Publishing Limited, volume 23. 331-402.

Del Negro, M. and F. Schorfheide. 2004. Priors from general equilibrium models for Vars. International Economic Review, 45(2): 643-673.

Del Negro, M. and F. Schorfheide. 2008. Forming priors for DSGE models (and how it affects the assessment of nominal rigidities). Journal of Monetary Economics, 55(7): 1191-1208.

Doan, T., R. Litterman, and C. Sims. 1984. Forecasting and conditional projection using realistic prior distributions. Econometric reviews, 3(1): 1-100.

Drèze, J.H. 1976. Bayesian limited information analysis of the simultaneous equations model. Econometrica, 44(5): 1045-75. 
Drèze, J.H. 1977. Bayesian regression analysis using poly-t densities. Journal of Econometrics, 6(3): 329-354.

Drèze, J.H. and J.F. Richard. 1983. Bayesian analysis of simultaneous equation systems. In Z. Griliches and M.D. Intriligator (eds.), Handbook of Econometrics, Elsevier, Handbook of Econometrics, volume 1, chapter 9. 517-598.

Durham, G. and J. Geweke. 2013. Adaptive sequential posterior simulators for massively parallel computing environments. Working Paper Series 9, Economics Discipline Group, UTS Business School, University of Technology, Sydney.

Escobar, M.D. and M. West. 1995. Bayesian density estimation and inference using mixtures. Journal of the american statistical association, 90(430): 577-588.

Ferguson, Thomas S. 1973. A Bayesian analysis of some nonparametric problems. The annals of statistics: 209-230.

Fernandez, C., E. Ley, and M.F.J. Steel. 2001. Model uncertainty in cross-country growth regressions. Journal of Applied Econometrics, 16(5): 563-576.

Fernandez-Villaverde, J. and J.F. Rubio-Ramirez. 2007. Estimating macroeconomic models: A likelihood approach. The Review of Economic Studies, 74(4): 1059-1087.

Fernandez-Villaverde, J. and J.F. Rubio-Ramirez. 2008. How structural are structural parameters? NBER Chapters: 83-137.

Fisher, R.A. 1912. On an absolute criterion for fitting frequency curves. Messenger of Mathmatics, 41: $155-160$.

Fisher, R.A. 1922. On the mathematical foundations of theoretical statistics. Philosophical Transactions of the Royal Society, A(222): 309-368.

Fisher, R.A. 1973. Statistical methods and scientific inference Hafner. New York.

Florens, J.P. and A. Simoni. 2012. Nonparametric estimation of an instrumental regression: a quasiBayesian approach based on regularized posterior. Journal of Econometrics, 170(2): 458-475.

Fruehwirth-Schnatter, S. 1994. Data augmentation and dynamic linear models. Journal of Time Series Analysis, 15(2): 183-202.

Gelfand, A.E. and D.K. Dey. 1994. Bayesian model choice: asymptotics and exact calculations. Journal of the Royal Statistical Society. Series B (Methodological): 501-514.

Gelfand, A.E. and A.F.M. Smith. 1990. Sampling-based approaches to calculating marginal densities. Journal of the American Statistical Association, 85(410): 398-409.

Geman, S. and D. Geman. 1984. Stochastic relaxation, Gibbs distributions, and the Bayesian restoration of images. IEEE Transactions on Pattern Analysis and Machine Intelligence, (6): 721-741.

George, E.I. and R.E. McCulloch. 1993. Variable selection via Gibbs sampling. Journal of the American Statistical Association, 88(423): 881-889.

Gerlach, R.C.C. and R. Kohn. 2000. Efficient Bayesian inference for dynamic mixture models. Journal of the American Statistical Association, 95(451): 819-828. 
Geweke, J. 1989. Bayesian inference in econometric models using Monte carlo integration. Econometrica, 57(6): 1317-39.

Geweke, J. 2005. Contemporary Bayesian econometrics and statistics. Wiley.

Geweke, J. 2010. Complete and incomplete econometric models. Princeton University Press.

Geweke, J. and G. Amisano. 2010. Comparing and evaluating Bayesian predictive distributions of asset returns. International Journal of Forecasting, 26(2): 216-230.

Geweke, J. and G. Amisano. 2011. Hierarchical Markov normal mixture models with applications to financial asset returns. Journal of Applied Econometrics, 26(1): 1-29.

Geweke, J.F., G. Koop, and H.K. Van Dijk (eds.). 2011. The Oxford handbook of Bayesian econometrics. Oxford University Press.

Giordani, P. and R. Kohn. 2008. Efficient Bayesian inference for multiple change-point and mixture innovation models. Journal of Business 83 Economic Statistics, 26(1).

Goldberger, A.S. 1972. Structural equation methods in the social sciences. Econometrica, 40(6): 9791001.

Gordon, N.J., D.J. Salmond, and A.F.M. Smith. 1993. Novel approach to nonlinear/non-Gaussian Bayesian state estimation. In IEE Proceedings F (Radar and Signal Processing). IET, volume 140, $107-113$.

Griffin, J.E. and M.F.J. Steel. 2004. Semiparametric Bayesian inference for stochastic frontier models. Journal of Econometrics, 123(1): 121-152.

Haavelmo, T. 1944. The probability approach in econometrics. Cowles Foundation paper 4, Cowles Commission. Reprinted in Econometrica (1944) 12: 1-118.

Hammersley, J.M. and D.C. Handscomb. 1964. Monte carlo methods. Chapman \& Hall, London.

Hansen, K., V. Singh, and P. Chintagunta. 2006. Understanding store-brand purchase behavior across categories. Marketing Science, 25(1): pp. 75-90.

Harvey, A. C. 1990. Forecasting, structural time series models and the Kalman filter. Cambridge university press.

Harvey, A.C., T.M. Trimbur, and H.K. Van Dijk. 2007. Trends and cycles in economic time series: A Bayesian approach. Journal of Econometrics, 140(2): 618-649.

Hastings, W.K. 1970. Monte Carlo sampling methods using Markov chains and their applications. Biometrika, 57(1): 97-109.

Hirano, K. 2002. Semiparametric Bayesian inference in autoregressive panel data models. Econometrica, 70(2): 781-799.

Hirsch, J.E. 2005. An index to quantify an individual's scientific research output. Proceedings of the National academy of Sciences of the United States of America, 102(46): 165-169.

Hood, W.C. and T.C. Koopmans (eds.). 1953. Studies in Econometric Method. John Wiley \& Sons. Cowles Commission Monograph No. 14. 
Hoogerheide, Lennart, Anne Opschoor, and Herman K. Van Dijk. 2012. A class of adaptive importance sampling weighted EM algorithms for efficient and robust posterior and predictive simulation. Journal of Econometrics, 171(2): 101-120.

Hoogerheide, L.F., J.F. Kaashoek, and H.K. Van Dijk. 2007. On the shape of posterior densities and credible sets in instrumental variable regression models with reduced rank: An application of flexible sampling methods using neural networks. Journal of Econometrics, 139(1): 154-180.

Hurwicz, L. 1950. Bayes and minimax interpretation of the maximum likelihood estimation criterion. Cowles Commission Discussion Paper Economics 352, Cowles Commission.

Ishwaran, H. and J.S. Rao. 2005. Spike and slab variable selection: frequentist and Bayesian strategies. Annals of Statistics: 730-773.

Jacquier, E., N.G. Polson, and P.E. Rossi. 1994. Bayesian analysis of stochastic volatility models. Journal of Business \& Economic Statistics, 12(4): 371-389.

Jensen, M.J. 2004. Semiparametric Bayesian inference of long-memory stochastic volatility models. Journal of Time Series Analysis, 25(6): 895-922.

Jensen, M.J. and J.M. Maheu. 2010. Bayesian semiparametric stochastic volatility modeling. Journal of Econometrics, 157(2): 306-316.

Kadiyala, K.R. and S. Karlsson. 1997. Numerical methods for estimation and inference in Bayesian VAR-models. Journal of Applied Econometrics, 12(2): 99-132.

Kim, S., N. Shephard, and S. Chib. 1998. Stochastic volatility: Likelihood inference and comparison with ARCH models. The Review of Economic Studies, 65(3): 361-393.

Kleibergen, F. and H.K. Van Dijk. 1993. Non-stationarity in GARCH models: A Bayesian analysis. Journal of Applied Econometrics, 8: 41-61.

Kleibergen, F. and H.K. Van Dijk. 1994. On the shape of the likelihood/posterior in cointegration models. Econometric Theory, 10(3/4): 514-551.

Kleibergen, F. and H.K. Van Dijk. 1998. Bayesian simultaneous equations analysis using reduced rank structures. Econometric Theory, 14(6): 701-743.

Kloek, T. and H.K. Van Dijk. 1975. Bayesian estimates of equation system parameters: An unorthodox application of Monte Carlo. Erasmus University.

Kloek, T. and H.K. Van Dijk. 1978. Bayesian estimates of equation system parameters: An application of integration by Monte Carlo. Econometrica, 46(1): 1-19.

Koop, G., D.J. Poirier, and J.L. Tobias. 2007. Bayesian econometric methods, volume 7. Cambridge University Press.

Koopman, S.J. and J. Durbin. 2000. Fast filtering and smoothing for multivariate state space models. Journal of Time Series Analysis, 21(3): 281-296.

Koopmans, T. 1945. Statistical estimation of simultaneous economic relations. Journal of the American Statistical Association, 40(232): 448-466.

Koopmans, T.C. (ed.). 1950. Statistical inference in dynamic economic models. John Wiley \& Sons. Cowles Commission Monograph No. 10. 
Lancaster, T. 2004. An introduction to modern Bayesian econometrics. Blackwell Oxford.

Lindley, D.V. 1957. A statistical paradox. Biometrika, 44(1-2): 187-192.

Lindley, D.V. and G.M. El-Sayyad. 1968. The Bayesian estimation of a linear functional relationships. Journal of the Royal Statistical Society. Series B (Methodological): 190-202.

McCloskey, D.N. and S.T. Ziliak. 1996. The standard error of regressions. Journal of Economic Literature, $34(1): 97-114$.

McCulloch, R.E. and R.S. Tsay. 1994. Bayesian inference of trend- and difference-stationarity. Econometric Theory, 10(3-4): 596-608.

Metropolis, N., A.W. Rosenbluth, M.N. Rosenbluth, A.H. Teller, and E. Teller. 1953. Equation of state calculations by fast computing machines. The Journal of Chemical Physics, 21(6): 1087-1092.

Min, C. and A. Zellner. 1993. Bayesian and non-Bayesian methods for combining models and forecasts with applications to forecasting international growth rates. Journal of Econometrics, 56 (1-2): 89-118.

Neal, R.M. 2000. Markov chain sampling methods for Dirichlet process mixture models. Journal of computational and graphical statistics, 9(2): 249-265.

O'Hagan, A. 1995. Fractional Bayes factors for model comparison. Journal of the Royal Statistical Society. Series B (Methodological): 99-138.

Omori, Y., S. Chib, N. Shephard, and J. Nakajima. 2007. Stochastic volatility with leverage: Fast and efficient likelihood inference. Journal of Econometrics, 140(2): 425-449.

Paap, R. and H.K. Van Dijk. 1998. Distribution and mobility of wealth of nations. European Economic Review, 42(7): 1269-1293.

Park, T. and G. Casella. 2008. The Bayesian lasso. Journal of the American Statistical Association, 103(482): 681-686.

Phillips, P.C.B. 1991. To criticize the critics: An objective Bayesian analysis of stochastic trends. Journal of Applied Econometrics, 6(4): 333-364.

Pitt, M.K. and N. Shephard. 1999. Filtering via simulation: auxiliary particle filters. Journal of the American Statistical Association, 94(446): 590-599.

Poirier, D.J. 1989. A report from the battlefront. Journal of Business \& Economic Statistics, 7(1): $137-139$.

Poirier, D.J. 1992. A return to the battlefront. Journal of Business \& Economic Statistics, 10(4): 473-474.

Pratt, J.W., H. Raiffa, and R. Schlaifer. 1995. Introduction to statistical decision theory. MIT press.

Raiffa, H. and R. Schlaifer (eds.). 1961. Applied statistical decision theory. Harvard University Press.

Robert, C.P. and G. Casella. 2004. Monte Carlo statistical methods. Springer Verlaag.

Rossi, P.E., G.M. Allemby, and R. McCulloch. 2005. Bayesian statistics and marketing. Wiley Series in Probability and Statistics. 
Rossi, P.E., R.E. McCulloch, and G.M. Allenby. 1996. The value of purchase history data in target marketing. Marketing Science, 15(4): 321-340.

Rothenberg, T.J. 1963. A Bayesian analysis of simultaneous equation system. Econometric Institute Report 6315, Erasmus University Rotterdam.

Rothenberg, T.J. 1973. Efficient estimation with a priori information. Yale University Press. Cowles Commission Monograph No. 23.

Sargan, J.D. 1958. The estimation of economic relationships using instrumental variables. Econometrica: Journal of the Econometric Society, 26: 393-415.

Savage, L.J. 1961. The subjective basis of statistical practice. University of Michigan.

Schlaifer, R. 1959. Probability and statistics for business decisions: An introduction to magagerial economics under uncertainty. McGraw-Hill.

Schotman, P. and H.K. Van Dijk. 1991a. A Bayesian analysis of the unit root in real exchange rates. Journal of Econometrics, 49(1-2): 195-238.

Schotman, P.C. and H.K. Van Dijk. 1991b. On Bayesian routes to unit roots. Journal of Applied Econometrics, 6(4): 387-401.

Sethuraman, J. 1994. A constructive definition of Dirichlet priors. Statistica Sinica, 4: 639-650.

Sims, C. and T. Zha. 2006. Were there regime switches in U.S. monetary policy? American Economic Review, 96(1): 54-81.

Sims, C.A. 2007. Bayesian methods in applied econometrics, or, why econometrics should always and everywhere be Bayesian.

Sims, C.A. and H. Uhlig. 1991. Understanding unit rooters: A helicopter tour. Econometrica, 59(6): $1591-1599$.

Smets, F. and R. Wouters. 2003. An estimated dynamic stochastic general equilibrium model of the Euro area. Journal of the European economic association, 1(5): 1123-1175.

Smets, F. and R. Wouters. 2007. Shocks and frictions in US business cycles: A Bayesian DSGE approach. American Economic Review, 97(3): 586-606.

Strachan, R.W. and H.K. Van Dijk. 2013. Evidence on features of a DSGE business cycle model from Bayesian model averaging. International Economic Review, 54(1): 385-402.

Tanner, M.A. and W.H. Wong. 1987. The calculation of posterior distributions by data augmentation. Journal of the American Statistical Association, 82: 528-540.

The Economist. 2004. Sugnifying nothing? Economics focus. January 31st, pp. 63.

Tierney, L. 1994. Markov chains for exploring posterior distributions. the Annals of Statistics: 17011728.

Van Dijk, H.K. 2013. Bridging two key issues in Bayesian inference: The relationship between the Lindley Paradox and non-elliptical credible sets. In N. Singpurwalla, P. Dawid, and A. O'Hagan (eds.), Festschrift for Dennis Lindley's ninetienth birthday. 
Van Dijk, H.K. and T. Kloek. 1980. Further experience in Bayesian analysis using Monte Carlo integration. Journal of Econometrics, 14(3): 307-328.

Van Dijk, H.K. and T. Kloek. 1985. Experiments with some alternatives for simple importance sampling in Monte Carlo integration. In J.M. Bernardo, M. Degroot, D. Lindley, and A.F.M. Smith (eds.), Bayesian Statistics, North-Holland, Amsterdam, volume 2. 511-530.

Van Eck, N.J and L. Waltman. 2010. Software survey: VOSviewer, a computer program for bibliometric mapping. Scientometrics, 84(2): 523-538.

Walker, S.G. 2007. Sampling the Dirichlet mixture model with slices. Communications in StatisticsSimulation and Computation, 36(1): 45-54.

Waltman, L., N.J. Van Eck, and E.C.M Noyons. 2010. A unified approach to mapping and clustering of bibliometric networks. Journal of Informetrics, 4(4): 629-635.

Wright, J.H. 2008. Bayesian model averaging and exchange rate forecasts. Journal of Econometrics, 146(2): 329-341. Honoring the research contributions of Charles R. Nelson.

Zellner, A. 1971. An introduction to Bayesian inference in econometrics. Wiley.

Zellner, A., T. Ando, N. Baştürk, L. Hoogerheide, and H. K. Van Dijk. 2014. Bayesian analysis of instrumental variable models: Acceptance-rejection within Direct Monte Carlo. Econometric Reviews, 33: $3-35$.

Zellner, A., L. Bauwens, and H.K. Van Dijk. 1988. Bayesian specification analysis and estimation of simultaneous equation models using Monte Carlo methods. Journal of Econometrics, 38(1-2): 39-72. 


\section{Appendix}

\section{A Bayesian Papers in Leading Journals}

Table A.1: Percentages of pages devoted to Bayesian papers in leading journals

\begin{tabular}{|c|c|c|c|c|c|c|c|c|c|c|}
\hline Years & RES & ReStat & Ectra & IER & $\mathrm{ET}$ & MS & $\mathrm{ER}$ & $\mathrm{JE}$ & JAE & JBES \\
\hline 1978 & 0.00 & 0.00 & 2.92 & 0.00 & - & - & - & 6.27 & - & - \\
\hline 1979 & 0.00 & 0.00 & 0.25 & 1.77 & - & - & - & 4.76 & - & - \\
\hline 1980 & 0.00 & 0.00 & 0.00 & 5.12 & - & - & - & 7.17 & - & - \\
\hline 1981 & 1.32 & 0.00 & 0.00 & 1.87 & - & - & - & 5.70 & - & - \\
\hline 1982 & 0.00 & 0.97 & 0.75 & 1.60 & - & 0.00 & 12.46 & 10.69 & - & - \\
\hline 1983 & 0.00 & 0.00 & 0.00 & 0.00 & - & 10.00 & 1.50 & 12.31 & - & 11.14 \\
\hline 1984 & 0.00 & 1.81 & 1.40 & 0.00 & - & 0.00 & 44.93 & 10.97 & - & 4.32 \\
\hline 1985 & 0.00 & 1.52 & 1.11 & 3.17 & 0.00 & 5.61 & 29.86 & 15.64 & - & 6.95 \\
\hline 1986 & 5.54 & 0.00 & 0.00 & 0.00 & 0.00 & 0.00 & 0.00 & 4.89 & 4.08 & 15.80 \\
\hline 1987 & 0.00 & 1.47 & 0.00 & 0.00 & 0.65 & 0.00 & 0.00 & 0.00 & 5.40 & 15.47 \\
\hline 1988 & 0.00 & 0.00 & 0.00 & 2.38 & 0.00 & 0.00 & 0.00 & 13.57 & 0.00 & 5.52 \\
\hline 1989 & 0.00 & 2.83 & 2.73 & 1.58 & 0.00 & 0.00 & 0.00 & 5.21 & 3.84 & 3.17 \\
\hline 1990 & 0.00 & 1.10 & 0.00 & 0.00 & 0.00 & 4.66 & 0.00 & 3.66 & 0.00 & 5.39 \\
\hline 1991 & 1.24 & 0.00 & 0.98 & 2.41 & 0.00 & 0.00 & 0.00 & 24.91 & 29.92 & 27.73 \\
\hline 1992 & 0.00 & 0.00 & 1.63 & 0.00 & 2.92 & 0.00 & 3.49 & 7.95 & 5.47 & 18.62 \\
\hline 1993 & 0.00 & 0.00 & 1.83 & 0.00 & 0.00 & 0.00 & 0.00 & 20.65 & 11.79 & 7.82 \\
\hline 1994 & 0.00 & 5.07 & 1.76 & 0.00 & 33.13 & 0.00 & 10.75 & 9.47 & 3.10 & 24.60 \\
\hline 1995 & 0.00 & 5.83 & 0.00 & 2.36 & 0.00 & 1.57 & 0.00 & 14.88 & 0.00 & 4.87 \\
\hline 1996 & 0.00 & 0.65 & 3.35 & 0.00 & 8.87 & 18.53 & 0.00 & 13.34 & 5.34 & 8.60 \\
\hline 1997 & 0.00 & 0.00 & 0.00 & 1.41 & 0.00 & 5.88 & 0.00 & 10.91 & 15.75 & 9.07 \\
\hline 1998 & 3.79 & 8.01 & 0.00 & 3.32 & 8.74 & 3.76 & 5.48 & 13.04 & 10.09 & 11.37 \\
\hline 1999 & 0.00 & 2.98 & 2.90 & 0.00 & 0.00 & 14.38 & 32.67 & 5.98 & 10.45 & 12.82 \\
\hline 2000 & 0.00 & 1.41 & 0.00 & 0.00 & 2.11 & 12.56 & 0.00 & 18.05 & 16.40 & 26.41 \\
\hline 2001 & 0.00 & 2.69 & 4.19 & 6.88 & 0.69 & 0.00 & 8.04 & 4.40 & 7.17 & 13.59 \\
\hline 2002 & 1.96 & 0.00 & 0.75 & 0.00 & 4.25 & 19.71 & 4.03 & 9.19 & 8.81 & 4.20 \\
\hline 2003 & 0.00 & 0.00 & 3.70 & 4.41 & 0.00 & 11.76 & 2.92 & 15.65 & 10.49 & 20.93 \\
\hline 2004 & 0.00 & 0.00 & 5.94 & 4.61 & 0.00 & 12.68 & 0.00 & 16.57 & 6.73 & 21.22 \\
\hline 2005 & 5.15 & 1.65 & 0.00 & 0.00 & 2.72 & 7.25 & 0.00 & 11.92 & 16.23 & 11.67 \\
\hline 2006 & 2.47 & 0.00 & 1.56 & 0.00 & 0.00 & 15.03 & 31.43 & 6.14 & 12.37 & 11.44 \\
\hline 2007 & 4.14 & 0.00 & 4.35 & 2.27 & 2.78 & 16.74 & 56.83 & 12.45 & 1.57 & 14.40 \\
\hline 2008 & 0.00 & 0.00 & 0.00 & 0.00 & 3.07 & 23.15 & 9.87 & 10.62 & 22.73 & 20.32 \\
\hline 2009 & 0.00 & 0.00 & 4.98 & 8.54 & 1.06 & 0.00 & 4.13 & 7.28 & 24.65 & 10.18 \\
\hline 2010 & 5.85 & 0.00 & 0.90 & 0.00 & 0.86 & 22.16 & 9.44 & 7.06 & 24.22 & 21.97 \\
\hline 2011 & 1.91 & 1.30 & 0.00 & 5.35 & 1.75 & 35.17 & 14.29 & 6.75 & 13.57 & 4.82 \\
\hline 2012 & 0.00 & 4.49 & 2.10 & 4.82 & 0.00 & 13.65 & 6.70 & 13.44 & 18.32 & 13.42 \\
\hline 1978-2012 & 0.95 & 1.25 & 1.43 & 1.82 & 2.63 & 8.20 & 9.32 & 10.33 & 10.68 & 12.93 \\
\hline
\end{tabular}

The table presents the percentages of pages devoted to Bayesian papers in the journals for each year and average percentages for the period 1978-2012. The table is an extension of Table 2 in Poirier (1992). The numbers in red correspond to years with special issues. Econometric Reviews, Econometric Theory, Journal of Applied Econometrics and Marketing Science did not exist before 1982, 1985, 1986 and 1982, respectively. Average numbers of Bayesian pages only include years for which the journal existed. Journal abbreviations are as in Figure 2. 
Table A.2: Citation information for papers in leading journals

\begin{tabular}{lrrrrrrrrrrr} 
& Total & RES & ReStat & IER & Ectra & ET & MS & ER & JAE & JE & JBES \\
\hline All papers & & & & & & & & & & \\
Number of citations & 64041 & 2796 & 3075 & 2427 & 5586 & 1718 & 7399 & 3252 & 7548 & 19712 & 10527 \\
Number of cited papers & 912 & 15 & 29 & 28 & 31 & 31 & 117 & 49 & 116 & 320 & 175 \\
Number of Bayesian papers & 978 & 15 & 29 & 28 & 31 & 33 & 119 & 67 & 121 & 328 & 205 \\
Papers with at least 100 citations & & & & & & & & & & \\
Number of citations & 43402 & 2471 & 2273 & 1677 & 4724 & 1103 & 4499 & 2487 & 5063 & 12192 & 6913 \\
Number of Bayesian papers & 163 & 6 & 9 & 6 & 12 & 7 & 21 & 4 & 20 & 53 & 25 \\
Papers with at least 400 citations & & & & & & & & & & \\
Number of citations & 17851 & 1872 & 752 & 0 & 3238 & 409 & 897 & 2382 & 1639 & 3277 & 3385 \\
Number of Bayesian papers & 28 & 2 & 1 & 0 & 5 & 1 & 2 & 3 & 3 & 6 & 5 \\
\hline
\end{tabular}

The table presents citation information for each leading journal and for the leading journals jointly. Total number of cited papers is based on papers which are cited at least once. Journal abbreviations are as in Figure 2 .

Table A.3: Average number of citations for papers in leading journals for 5-year intervals

\begin{tabular}{lrrrrrrrrrr} 
Years & RES & ReStat & Ectra & IER & ET & MS & ER & JAE & JBES & JE \\
\hline All papers & & & & & & & & & & \\
$1978-1982$ & 2 & 6 & 128 & 79 & - & 0 & 6 & - & 265 & - \\
$1983-1987$ & 11 & 48 & 21 & 1 & 0 & 102 & 197 & 157 & 178 & 339 \\
$1988-1992$ & 6 & 110 & 312 & 26 & 3 & 22 & 1 & 139 & 335 & 113 \\
$1993-1997$ & 0 & 108 & 120 & 17 & 241 & 225 & 8 & 227 & 886 & 689 \\
$1998-2002$ & 291 & 283 & 259 & 125 & 72 & 413 & 157 & 386 & 950 & 422 \\
$2003-2007$ & 197 & 22 & 187 & 126 & 12 & 464 & 261 & 247 & 956 & 378 \\
$2008-2012$ & 52 & 39 & 90 & 111 & 15 & 253 & 26 & 447 & 372 & 165 \\
$1978-2012$ & 80 & 88 & 160 & 69 & 61 & 239 & 105 & 280 & 563 & 351 \\
Papers with at least 100 citations & & & & & & & & \\
$1978-1982$ & 0 & 0 & 119 & 70 & - & 0 & 0 & - & 107 & - \\
$1983-1987$ & 0 & 34 & 0 & 0 & 0 & 89 & 188 & 115 & 77 & 272 \\
$1988-1992$ & 0 & 94 & 306 & 0 & 0 & 21 & 0 & 85 & 187 & 52 \\
$1993-1997$ & 0 & 64 & 114 & 0 & 174 & 194 & 0 & 193 & 574 & 568 \\
$1998-2002$ & 291 & 241 & 245 & 100 & 47 & 357 & 134 & 297 & 678 & 250 \\
$2003-2007$ & 183 & 22 & 118 & 119 & 0 & 219 & 176 & 164 & 679 & 241 \\
$2008-2012$ & 20 & 0 & 43 & 46 & 0 & 21 & 0 & 227 & 136 & 0 \\
$1978-2012$ & 71 & 65 & 135 & 48 & 39 & 145 & 80 & 188 & 348 & 230 \\
\hline
\end{tabular}

The table presents the average citation numbers of the Bayesian papers in the journals for the 5 year periods for all papers in leading journals (top panel) and a subset of papers with at least 100 citations (bottom panel). The table is an extension of Table 2 in Poirier (1992). Note that the period of observation is different for the following journals: Econometric Theory did not exist before 1985. Therefore the mean for the period 1983-1987 is taken over the periods 1985-1987. Journal of Applied Econometrics did not exist before 1986. Therefore, the mean over the years 1983-1987 is equal to the mean for 1986-1987. Econometric Reviews did not exist before 1982. Therefore, the mean for the period 1978-1982 is equal to the value in 1982. Marketing Science did not exist before 1982. So, the mean for the period 1978-1982 is equal to the value in 1982. Journal abbreviations are as in Figure 2. 


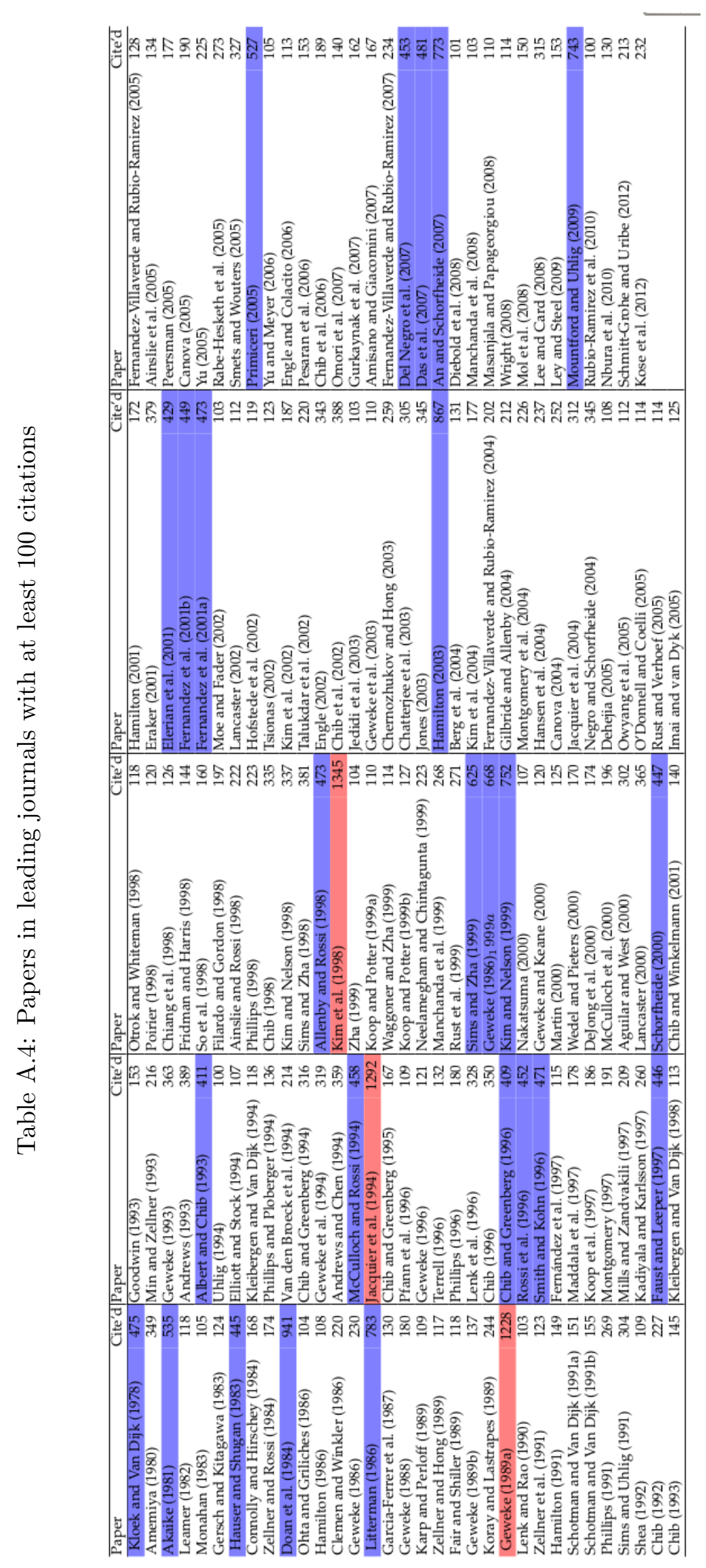

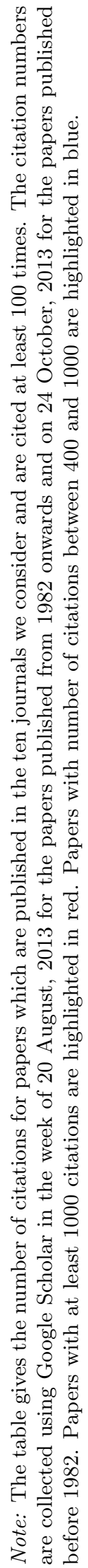

